\title{
Towards an Enumerative Geometry of the Moduli Space of Curves
}

\author{
David Mumford
}

Dedicated to Igor Shafarevitch on his 60th birthday

\section{Introduction}

The goal of this paper is to formulate and to begin an exploration of the cnumerative geometry of the set of all curves of arbitrary genus $g$. By this we mean setting up a Chow ring for the moduli space $M_{g}$ of curves of genus $g$ and its compactification $\bar{M}_{g}$, defining what seem to be the most important classes in this ring and calculating the class of some geometrically important loci in $\bar{M}_{g}$ in terms of these classes. We take as a model for this the enumerative geometry of the Grassmannians. Here the basic classes are the Chern classes of the tautological or universal bundle that lives over the Grassmannian, and the most basic cycles are the loci of linear spaces satisfying various Schubert conditions: the so-called Schubert cycles. However, since Ilarris and I have shown that for $g$ large, $\mathcal{M}_{g}$ is not unirational $[\mathrm{II}-\mathrm{M}]$ it is not possible to expect that $\mathcal{M}_{y}$ has a decomposition into elementary cells or that the Chow ring of $\mathcal{M}_{g}$ is as simple as that of the Grassmannian. But in the other direction, J. Harer [Ha] and E. Miller [Mi] have strong results indicating that at least the low dimensional homology groups of $M_{g}$ behave nicely. Moreover, it appers that many geometrically natural cycles are all expressible in terms of a small number of basic classes.

More specifically, the paper is divided into 3 parts. The goal of the first part is to define an intersection product in the Chow group of $\bar{M}_{g}$. The problern is that due to curves with automorphisms, $\bar{M}_{g}$ is singular, but in a mild way. In fact it is a " $Q$-variety", locally the quotient of a smooth variety by a finite group. If it were globally the quotient of a smooth variety by a finite group, it would be casy to define a product in $A \cdot\left(\bar{M}_{g}\right) \otimes \mathbb{Q}$. Instead we have used the fact that $\bar{M}_{g}$ is globally the quotient of a Cohen-Macaulay variety by a finite group, plus many of the ideas of Fulton and MacPherson, and especially a strong use of both the Grothendieck and Baum-FultonMacl'herson forms of the Riemann-Roch thcorem to achieve this goal. To 
handle an arbitrary $Q$-varicty, Gillet has proposed using higher $K$-theory $\left(I^{n}\left(K^{n}\right) \cong A^{n}\right)$ and this may well be the right technique.

The goal of the second part is to introduce a sequence of "tautological" classes $\kappa_{i} \in A^{i}\left(\bar{M}_{g}\right) \otimes \mathbb{Q}$, derive some relations between them, and calculate the fundamental class of certain subvarieties, such as the hyperelliptic locus, in terms of them. Again the Grothendieck Riemann-Roch theorem is one of the main tools. Some of these results have been found independently by $\mathrm{F}$. Miller [Mi], and it seems reasonable to guess, in view of the results of Harer and Miller (op. cit.), that in low codimensions $H^{i}\left(M_{g}\right) \otimes \mathbb{Q}$ is a poly nomial ring in the $\kappa_{i}$.

Finally, to make the whole theory concrete, we work out $\Lambda\left(\bar{M}_{2}\right)$ completely in Part III. An interesting corollary is the proof, as a consequence of general results only, that $\mathcal{M}_{2}$ is affine. It seems very worthwhile to work out $A^{\cdot}\left(\bar{M}_{g}\right)$ or $I^{\cdot}\left(\mathcal{M}_{g}\right)$ for other small values of $g$, in order to get some fecling for the properties of these rings and their relation to the geometry of $\bar{M}_{g}$. The techniques of Atiyah-Bott [A-B] may be very useful in doing this.

\section{Part I: Defining a Chow Ring of the Moduli Space}

\section{§1. Fulton's Operational Chow Ring}

If $X$ is any quasi-projective varicty, Fulton and Fulton-MacPherson have defined in two papers ([F1], p. 157, [F-M], p. 92) two procedures to attach to $X$ a kind of Chow cohomology theory: a ring-valued contravariant functor. We combine here the 2 definitions taking the simplest parts of them in a way that is adequate for our applications. The theory also becomes substantially simpler if we take the coefficients for our cycles to be $Q$, and we assume that $\operatorname{char} k=0$. This is the case we are interested in, so we will restrict ourselves to this case henceforth. We call the resulting ring op $\Lambda(X)$. To form this ring, take:

$$
\begin{aligned}
\text { generators : } & \text { elts }(f, \alpha), \\
& f: X \rightarrow Y \text { a morphism } \\
& Y \text { smooth, quasi-projective } \\
& \alpha \text { a cycle on } Y
\end{aligned}
$$


relations: $(f, \alpha) \sim 0$ if, for all $g: Z \rightarrow X$, we have: $(f \circ g)^{*} \alpha$ rationally equivalent to 0 on $Z$ via the induced map

$$
\begin{gathered}
A^{k}(Y) \stackrel{(f \circ g)^{*}}{\longrightarrow} A_{n-k}(Z) \\
(k=\operatorname{codim} \text { of } \alpha, n=\operatorname{dim} \text { of } Z)
\end{gathered}
$$

Equivalently, we may define

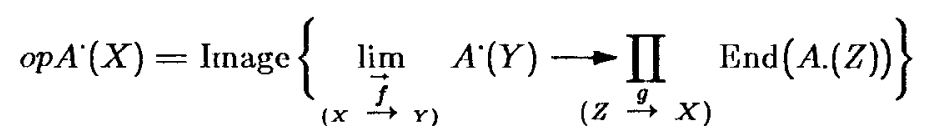

where the map is given by cap product

$$
A^{k}(Y) \times A_{l}(Z) \stackrel{\cap}{\longrightarrow} A_{l-k}(Z)
$$

(cf. $[\mathrm{F} 1], \S 2)$. This makes it clear that op $A^{*}$ is a ring and a contravarient functor and that $o p A(X)$ acts on $A .(X)$ by cap product. If $X$ is smooth, then $o p A^{\prime}(X) \cong A^{\prime}(X)$.

Moreover, as in l'ulton [F1], §3.2, for all, coherent sheaves $\mathcal{F}$ with finite projective resolutions, we can define the Chern classes $c_{k}(\mathcal{F}) \in o p A^{k}(X)$, (by resolution of $\mathcal{F}$, twisting and pull-back of Schubert cycles from maps of $X$ to Grassmannians).

Using a resolution of $X$, we can give a very simple description of the relations in $o p A^{\prime}(X)$ :

Proposition 1.1. If $\pi: \tilde{X} \rightarrow X$ is a resolution of $X$, then

$$
(f, \alpha) \sim 0 \Leftrightarrow(f \circ \pi)^{*}(\alpha)=0 \quad \text { in } A^{k}(\tilde{X}),
$$

i.e.,

$$
o p A^{\cdot}(X) \subset \Lambda^{\cdot}(\tilde{X})
$$

Proof. We must show that if

$$
g: Z \rightarrow X
$$

is any test morphism, then $l(f \circ \pi)^{*} \alpha$ rationally equivalent to 0 on $\tilde{X}$ implies $l^{\prime}(f \circ g)^{*} \alpha$ rationally equivalent to 0 on $Z$ for some $l^{\prime}$. But by taking a 
suitable subvariety of $Z \times_{X} \tilde{X}$ we get a diagram

$$
\begin{aligned}
& \tilde{Z} \stackrel{\tilde{g}}{\rightarrow} \tilde{X} \\
& p \downarrow \downarrow \pi \\
& Z \stackrel{g}{\rightarrow} X \stackrel{f}{\rightarrow} Y
\end{aligned}
$$

where $p$ is proper, surjective, gencrically finite of degree $l^{\prime \prime}$. Therefore

$$
\begin{aligned}
l^{\prime \prime}(f \circ g)^{*} \alpha & =p_{*}\left((f \circ g \circ p)^{*} \alpha\right) \\
& =p_{*}\left(\tilde{g}^{*}\left((f \circ \pi)^{*} \alpha\right)\right)
\end{aligned}
$$

hence

$$
l . l^{\prime \prime}(f \circ g)^{*} \alpha=p_{*}\left(\tilde{g}^{*}\left(l(f \circ \pi)^{*} \alpha\right)\right) \sim 0 .
$$

This uses the formula:

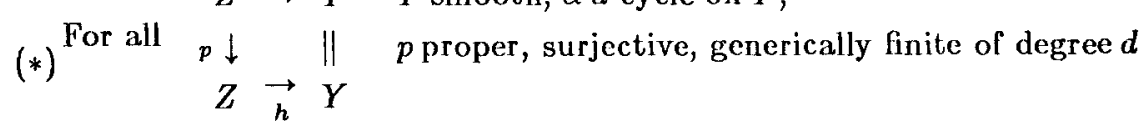

$$
\begin{aligned}
& \begin{aligned}
\tilde{Z} & \stackrel{\tilde{h}}{\rightarrow} \\
p \downarrow & \\
Z \underset{h}{\rightarrow} & Y
\end{aligned} \\
& p_{*} \tilde{h}^{*}(\alpha) \sim d h^{*}(\alpha) .
\end{aligned}
$$

(See [F1], §2.2, part 2 of lemma).

In fact, we can say more:

Proposition 1.2. In the situation of Prop. 1.1, the image of op $A^{k}(X)$ in $A^{k}(\tilde{X})$ is contained in the subgroup of $A^{k}(\tilde{X})$ generated by irreducible subvarieties $W$ of $\tilde{X}$ such that $W=\pi^{-1}(\pi(W))$.

Proof. Let $(f, \alpha)$ be a generator of op $A^{k}(X)$, where $f: X \rightarrow Y$ is a morphism and $Y$ is smooth, quasi-projective. Let $p=f \circ \pi$, and

$$
Y_{k}=\left\{y \in Y \mid \operatorname{dim} p^{-1}(y) \geq k\right\}
$$

Then use the moving lemma on $Y$ to represent $\alpha$ as a cycle $\sum n_{i} W_{i}$, whose components $W_{i}$ meet properly all the $Y_{k}$. Then each component $W_{i j}$ of $p^{-1}\left(W_{i}\right)$ incets the open set of $\tilde{X}$ where $\pi$ is an isomorphism and 
$p: \tilde{X} \longrightarrow p(\tilde{X})$ is equidimensional. Therefore, $p^{*} \alpha$ is represented by a combination of the $W_{i j}$ with suitable multiplicities and each $W_{i j}$ satisfies $W_{i j}=\pi^{-1}\left(\pi\left(W_{i j}\right)\right)$.

There is also another natural way to give generators for $o p A^{\cdot}(X)$ :

Proposition 1.3. Using rational coefficients in $K(X)$ too, the homomorphism

$$
\text { ch }: K(X) \longrightarrow o p A^{\prime}(X)
$$

is surjective, hence op $A^{\circ}(X)$ can be defined to be

$$
\text { Image } \begin{aligned}
{[K(X)} & \left.\rightarrow A^{\cdot}(\tilde{X})\right] \\
\mathcal{E} & \mapsto \operatorname{ch}\left(\pi^{*} \mathcal{E}\right)
\end{aligned}
$$

$(\pi: \tilde{X} \rightarrow X$ a resolution of $X)$.

Proof. It is well known that for any smooth quasi-projective $Z$, ch: $g r K(Z) \longrightarrow A^{\prime}(Z)$ is a graded isomorphism, hence taking the total Chern characters, ch: $K(Z) \rightarrow A^{\cdot}(Z)$ is also an isomorphism ${ }^{1}$. Therefore $c h: K(X) \rightarrow o p A^{\prime}(X)$ is surjective.

$o p A^{\cdot}(X)$ has a much subtler covariance for certain morphisms $f$, whose existence is tied up with the version of the Grothendieck-Riemann-Roch theorems for $o p A^{*}$. The result is this:

Theorem 1.4 (Fulton). Let $f: X \rightarrow Y$ be a projective local-completeintersection morphism. Define $T d_{f} \in o p A^{\prime}(X)$ in the usual way. Then there is a homomorphism

$$
f_{*}: o p A^{*}(X) \rightarrow o p A^{*}(Y)
$$

such that

${ }^{1}$ This sounds a bid odd, but it is perhaps clarified by the observation that for any rank $r$ and dimension $n$, there are universal polynomials $P_{k}$ such that for all vector bundles $\varepsilon$ of rank $r$ on $n$-dimensional varieties,

$$
c_{k}(\varepsilon)=P_{k}\left(\operatorname{ch} \mathcal{E}, \operatorname{ch} \Lambda^{2} \mathcal{E}, \ldots, \operatorname{ch} \Lambda^{r} \mathcal{E}\right)
$$

where ch $\mathcal{E}$ is the total Chern character, and these elements lie in any cohomology ring with the usual Chern formalism and rational coeffcients. 
1) for all cartesian diagrams

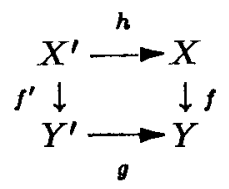

and all $\alpha \in A .\left(Y^{\prime}\right), \beta \in o p A^{\prime}(X)$

$$
f_{*} \beta \cap \alpha=f_{*}^{\prime}\left(\beta \cap[f]_{Y^{\prime}}(\alpha)\right) \text { in } A .\left(Y^{\prime}\right)
$$

$\left([f]_{Y}\right.$, defined as in Fulton-MacPherson [F-M], p. 95).

2) for all locally free sheaves $\mathcal{E}$ on $X$,

$$
\operatorname{ch}\left(f_{!} \mathcal{E}\right)=f_{*}\left(\operatorname{ch} \mathcal{E} \cdot T d_{f}\right) .
$$

This is proven in Fulton [F2], Ch.18: here op $A^{\circ}$ is a possibly larger Chow Ring in which (1) is the definition of $f_{*}$. In this ring (2) is proven, and (2) shows that $f_{*} \beta$ actually lies in the subring op $A^{*}$ considered here.

\section{§2. $Q$-Varieties and $\bar{M}_{g}$}

The moduli space $\overline{\mathcal{M}}_{g}$ of stable curves is an example of a variety which is locally in the étale topology a quotient of a smooth variety by a finite group. The approach we take to defining a Chow ring for $\bar{M}_{g}$ is best studied in this more general context. Because these varieties are quitc close to the objects introduced by Matsusaka [Ma], we shall call them quasi-projective $Q$-varieties. We define a quasi-projective $Q$-variety to be:

1) a quasi-projective variety $X$,

2) a finite atlas of charts:

$$
p_{\alpha}\left(\begin{array}{c}
X_{\alpha} \\
\downarrow \\
X_{\alpha} / G_{\alpha} \\
\downarrow p_{\alpha}^{\prime} \\
X
\end{array}\right.
$$


where $p_{\alpha}^{\prime}$ is étalc, $G_{\alpha}$ is a finite group acting, faithfully on a quasi-projective smooth $X_{\alpha}$ and

$$
X=\bigcup_{\alpha}\left(\operatorname{Im} p_{\alpha}\right)
$$

3) The charts should be compatible in the sensc that for all $\alpha, \beta$, let

$$
X_{\alpha \beta}=\text { normalization of } X_{\alpha} \times_{X} X_{\beta} .
$$

Then the projections

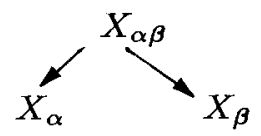

should be étale.

Herc, of course, a new chart can be added if it satisfies the compatibility conditions (3) with the old oncs. For any such $Q$-variety, we can normalize $X$ in a Galois extension of its function field $k(X)$ containing copies of the ficld extensions $k\left(X_{\alpha}\right)$ for all $\alpha$. This leads to a covering $p: \tilde{X} \rightarrow X$ with a group $G$ acting faithfully on $\tilde{X}$ and $X=\tilde{X} / G$. The fact that $k(\tilde{X}) \supset k\left(X_{\alpha}\right)$ leads to a factorization of $p$ locally:

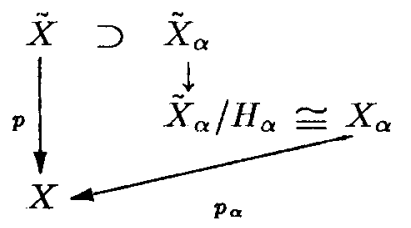

$$
\begin{aligned}
& \tilde{X}_{\alpha} \text { open in } \tilde{X} \text { stabilized by } \\
& \quad H_{\alpha}^{\prime} \subset G, \\
& H_{\alpha}=\text { normal subgroup of } H_{\alpha}^{\prime} \\
& \quad \text { with } G_{\alpha} \cong H_{\alpha}^{\prime} / H_{\alpha} .
\end{aligned}
$$

$Q$-varieties come in various different grades. The best ones are those such that for some atlas, $\tilde{X}$ can be chosen to be itself smooth. Not so nice, but still amcndable to the techniques we shall use are those where $\tilde{X}$ can be chosen to be Cohen-Macaulay. We call these $Q$-varjeties with a smooth global cover and with a Cohen-Macaulay global cover respectively.

Another important concept is that of a $Q$-sheaf on a $Q$-variety $X$. By this, we do not mean a coherent sheaf on $X$, but rather a family of coherent sheaves $\mathcal{F}_{\alpha}$ on $X_{\alpha}$, plus isomorphisms

$$
F_{\alpha} \otimes o_{X_{\alpha}} o_{X_{\alpha \beta}} \cong F_{\beta} \otimes o_{X_{\beta}} o_{X_{\alpha \beta}}
$$

compatible on the triple overlaps. Notc that such a coherent sheaf pulls back by tensor product to a family of coherent sheaves on $\tilde{X}_{\alpha}$, which 
glue together to one coherent sheaf $\tilde{f}$ on $\tilde{X}$ on which $G$ acts. Therefore, equivalently we can define a coherent sheaf on the $Q$-variety $X$ to be a coherent $G$-sheaf $\tilde{\mathcal{F}}$ on $\tilde{X}$ such that for all $\alpha,\left.\tilde{\mathcal{F}}\right|_{\tilde{X}_{\alpha}}$ with its $H_{\alpha}^{\prime}$-action is the pull-back of a coherent sheaf on $X_{\alpha}$. The importance of $\tilde{X}$ being Cohen-Macaulay is illustrated by the simple fact:

Proposition 2.1. If $\tilde{X}$ is Cohen-Macaulay, then for any coherent sheaf $\mathcal{F}$ on the $Q$-variety $X, \tilde{\mathcal{F}}$ has a finite projective resolution.

Proof. In fact, if $\tilde{X}$ is Cohen-Macaulay and $X_{\alpha}$ is smooth, $\tilde{X}_{\alpha} \rightarrow X_{\alpha}$ is flat. But $\exists_{\alpha}$ has a finite projective resolution, so this resolution pulls back to such a resolution for $\left.\tilde{\mathcal{F}}\right|_{\tilde{X}_{\alpha}}$.

Q.E.D.

Consider the case of the moduli space $\bar{M}_{g}$. Choose an integer $n \geq 3$ prime to the characteristic. Fix a free $\mathbb{Z} / n \mathbb{Z}$-module $V$ of rank $2 g$ with an alternating non-degenerate form

$$
e: V \times V \rightarrow \mu_{n}
$$

Fix, moreover, a flag of isotropic free submodules:

$$
\text { (0) } \subset V_{1} \subset V_{2} \subset \cdots \subset V_{g}=V_{g}^{\perp} \subset \cdots \subset V_{2}^{\perp} \subset V_{1}^{\perp} \subset V
$$

where $r k\left(V_{i}\right)=i$. Then for all stable curves $C$ of genus $g$, let $h$ be the sum of the genera of the components of its normalization. Then there is an isomorphism

$$
H^{1}(C, \mathbb{Z} / n \mathbb{Z}) \cong V_{g-h}^{\perp}
$$

such that the form

$$
H^{1} \times H^{1} \stackrel{\cup}{\rightarrow} H^{2} \stackrel{f^{\prime} \text { al class }}{\longrightarrow}-\mu_{n}
$$

corresponds to $e$. We may consider the auxiliary moduli space

$$
\begin{aligned}
\left(\overline{\mathcal{M}}_{g}^{(n, h)}\right)^{\prime}= & \text { set of pairs }(C, \phi), C \text { stable curve, } \\
& \phi: V_{g-h}^{\perp} \stackrel{\phi \text { injective }}{\longrightarrow} I I^{1}(C, \mathbb{Z} / n \mathbb{Z}) \text { a sympl. map }
\end{aligned}
$$


which can be constructed by standard arguments. Inside this space, define an open subset by:

$$
\begin{aligned}
\bar{M}_{g}^{(n, h)}= & \text { those pairs }(C, \phi) \text { such that every automorphism } \\
& \alpha: C \rightarrow C \text { fixes the submodule } \operatorname{Im} \phi \subset H^{1}(C, \mathbb{Z} / n \mathbb{Z}) \\
& \text { and, if } \alpha \neq 1_{C}, \text { then } \alpha \text { acts non-trivially on } \operatorname{Im} \phi .
\end{aligned}
$$

Since the pairs $(C, \phi)$ in this subset have no automorphisms, $\overline{\mathcal{M}}_{g}^{(n, h)}$ is smooth and represents the universal deformation space of any curve occuring in it. Note that every curve $C$ occurs in the space $\bar{M}_{g}^{(n, h)}$ such that $g+h=r k H^{1}(C)$ (see [D-M], Th. 1.13). Next consider the finite groups

$$
G=S p(V, \mathbb{Z} / n \mathbb{Z})
$$

$\cup$

$$
\left.H_{h}^{\prime}=\text { (stabilizer of } V_{g-h}\right)
$$

\section{$\cup$}

$$
\begin{aligned}
H_{h} & =\left(\begin{array}{l}
\text { elements which } \\
\text { act identically } \\
\text { on } V_{g-h}^{\perp}
\end{array}\right) \\
G_{h} & \left.=H_{h}^{\prime} / H_{h}=\text { (induced group of automorphisms of } V_{g-h}^{\perp}\right) .
\end{aligned}
$$

Then $G_{h}$ acts on $\bar{M}_{g}^{(n, h)}$ and we have canonical morphisms

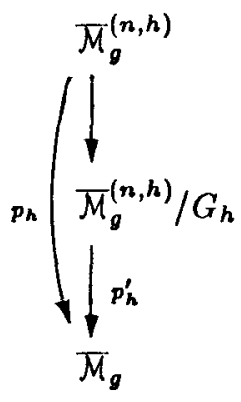


I claim that $p_{h}^{\prime}$ is ctale. In fact, if $(C, \phi) \in \bar{M}_{g}^{(n, h)}$, then $\operatorname{Aut}(C)$ can be identified with a subgroup of $G_{h}$ and formally near $[C, \phi]$, the isomorphism

$$
\overline{\mathcal{M}}_{g}^{(n, h)} \underset{\text { formal isom. }}{ } \quad \operatorname{Def}(C)
$$

commutes with the action of $\operatorname{Aut}(C)$.

Therefore we have a diagram

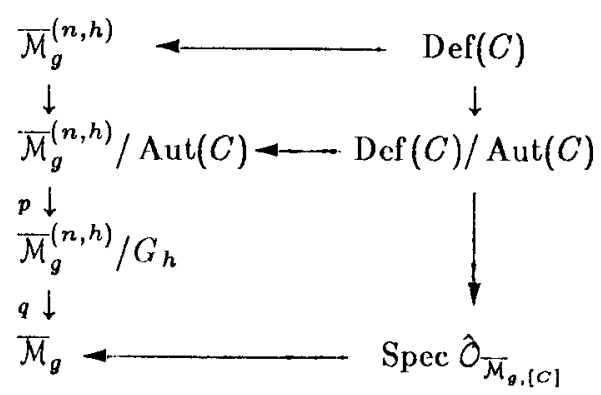

where the horizontal arrows are formal isomorphisms. Now Def $(C) / \Lambda \mathrm{ut}(C)$ i.e., Spce of the Aut $(C)$-invariants in the complete local ring representing the deformations of $C$, is isomorphic to Spec of the complete local ring of $\overline{\mathcal{M}}_{g}$ at $[\mathrm{C}]$. Thus the morphism indicated by $q \circ p$ in the diagram is étale at $\left[C^{\prime}, \phi\right]$. Therefore so is $q$.

This proves that the atlas $\left\{p_{h}: \bar{M}_{g}^{(n, h)} \rightarrow \overline{\mathcal{M}}_{g}\right\}$ puts a structure of $Q$-variety on $\overline{\mathcal{M}}_{g}$. In this setting, what is the variety $\tilde{X}$ dominating all the charts? This is

$$
\overline{\mathcal{M}}_{g}^{(n)}=\text { (normalization of } \overline{\mathcal{M}}_{g} \text { in this field extension } \overline{\mathcal{M}}_{g}^{(n, g)} \text { ). }
$$

Note that the full group $G$ acts on $\bar{M}_{g}^{(n)}$. Moreover, $\bar{M}_{g}^{(n, g)}$ is the open subset of $\bar{M}_{g}^{(n)}$ lying over the open set $\mathcal{M}_{g}$ of smnoth curves.

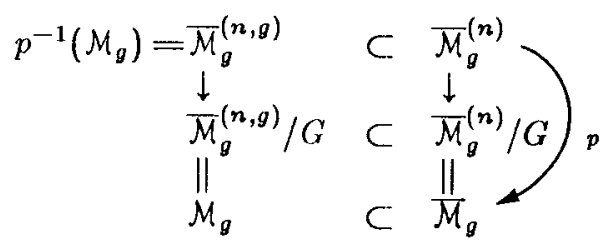


What we call $\overline{\mathcal{M}}_{g}^{(n, h)}$, for $h<g$, however, is recovered by dividing an open subset of $\bar{M}_{g}^{(n)}$ by $H_{h}$.

Life would be particularly simple if $\overline{\mathcal{M}}_{g}^{(n)}$ were smooth. However, it is not, nor is it known whether the normalization of $\overline{\mathcal{M}}_{g}^{(n)}$ in any finite extension field is smooth. However, fortunately $\bar{M}_{g}$ does have a Cohen-Macaulay global cover, because:

Proposition 2.2. $\quad \bar{M}_{g}^{(n, g)} \subset \overline{\mathcal{M}}_{g}^{(n)}$ is a toroidal embedding, i.e., $\overline{\mathcal{M}}_{g}^{(n)}$ is formally isomorphic to $A^{3 g-3}$ modulo an abelian group acting diagonally, and with $\bar{M}_{g}^{(n)}-\bar{M}_{g}^{(n, g)}$ isomorphic to the image of a union of coordinate hyperplanes in $\mathrm{A}^{3 g-3}$.

Proof. At every point of $\overline{\mathcal{M}}_{g}^{(n)}, \overline{\mathcal{M}}_{g}^{(n)}$ is a Galois covering of one of the smooth varieties $\overline{\mathcal{M}}_{g}^{(n, h)}$ with group $H_{h}$. Note that $H_{h}$ is abelian of order prime to char $(k)$. The covering is ramified only on $\overline{\mathcal{M}}_{g}^{(n, h)}-p_{h}^{-1}\left(\mathcal{M}_{g}\right)$. Since. $\bar{M}_{g}^{(n, h)}$ is formally the universal deformation space of some curve $C$, this is formally a ramified cover of $A^{39-3}$, ramified only in coordinate hyperplanes. But if char $(k) \not n$, the $n$-cyclic extensions $k\left[\left[x_{1}, \ldots, x_{3 g-3}\right]\right]$ ramified only over the ideals $\left(x_{i}\right)$ are all given by

$$
\left(\prod_{i \in I} x_{i}^{a_{i}}\right)^{1 / n}, \quad I \subset\{1, \ldots, 3 g-3\} .
$$

Thus the covering in hand is sandwiched between $k\left[\left[x_{1}, \ldots, x_{3 g-3}\right]\right]$ and $k\left[\left[x_{1}^{1 / n}, \ldots, x_{3 g-3}^{1 / n}\right]\right]$, hence, by Galois theory, is as described.

Corollary 2.3. $\quad \bar{M}_{g}^{(n)}$ is Cohen-Macaulay.

\section{$\S 2 b . Q$-Stacks}

Unfortunately, the concept of $Q$-variety, although adequate to deal with $\bar{M}_{g}, g \geq 3$, or with any moduli variety whose general object has no automorphisms, breaks down for $\bar{M}_{2}$ and $\bar{M}_{1,1}$ where the general object has automorphism group $\mathbb{Z} / 2 \not 2$. Consider, for instance, $\overline{\mathcal{M}}_{2}$. Let $\mathcal{M}_{2}^{o} \subset \bar{M}_{2}$ 
be the open set of smooth curves $C$ such that $\operatorname{Aut}(C) \cong \mathbb{Z} / 2 \mathbb{Z}$. Then, although $\mathcal{M}_{2}^{0}$ gives a local deformation space for its curves, it does not carry a universal family of curves. And if $\mathcal{M}_{2}^{\prime}$ is an étale cover of $\mathcal{M}_{2}^{o}$ carrying some family

$$
p: C^{\prime} \longrightarrow M_{2}^{\prime}
$$

the sheaf $\mathbb{E}=p_{*} \Omega_{C^{\prime} / \mathcal{M}^{\prime}}^{1}$ on $\mathcal{M}_{2}^{\prime}$ will not be a $Q$-sheaf. In fact, to compare $p_{1}^{*} \mathbb{E}, p_{2}^{*} \mathbb{E}$ on $\mathcal{M}_{2}^{\prime} \times \mathcal{M}_{2} \mathcal{M}_{2}^{\prime}$, we want an isomorphism of the 2 families

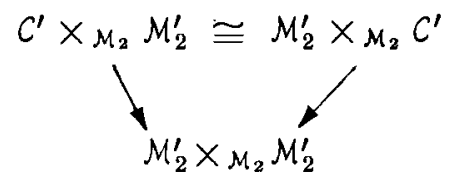

and although these families are fibrewise isomorphic, the isomorphism is not unique and may not globalize.

To deal with this, one must use some variant of the ideal of stack (cf. [D-M], §4). The most natural thing is to replace the normalization of $X_{\alpha} \times_{X} X_{\beta}$ by a scheme $X_{\alpha \beta}$ which nust be given as part of the data and cannot be derived from the rest. $X_{\alpha \beta}$ should map to $X_{\alpha} \times_{X} X_{\beta}$ and given $x \in X_{\alpha \beta}, y \in X_{\beta \gamma}$ with the same projection to $X_{\beta}$, a "composition" $x \circ y \in X_{\alpha \gamma}$ should be defined. A point $x \in X_{\alpha \beta}$ lying over $u \in X_{\alpha}$, $v \in X_{\beta}$ should be thought of as meaning an isomorphism from the object $C_{u}$ corresponding to $u$ to the object $C_{v}$ corresponding to $v$.

Definition 2.4. A Q-stack is a collection of quasi-projective varicties and morphisms:

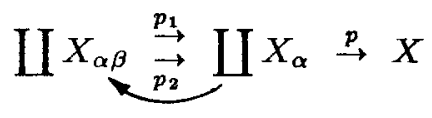

$X_{\alpha}, X_{\alpha \beta}$ smooth, $X$ normal, $p_{1}, p_{2}$ étale

$$
\begin{aligned}
\coprod X_{\alpha} & \rightarrow X \text { surjective } \\
X_{\alpha \beta} & \rightarrow X_{\alpha} \times X_{\beta} \text { surjective, finite } \\
p_{i} \circ \epsilon & =\text { identity }
\end{aligned}
$$


plus morphisms ${ }^{2}$ :

$$
\begin{aligned}
X_{\alpha \beta} X_{X_{\beta}} X_{\beta \gamma} & \stackrel{\circ}{\rightarrow} X_{\alpha \gamma} \\
X_{\alpha \beta} & \stackrel{-1}{\rightarrow} X_{\beta \alpha}
\end{aligned}
$$

making $\coprod X_{\alpha \beta}$ into a pseudo-group (i.e., $\circ$ is associative where defined, ${ }^{-1}$ is its inverse and $\epsilon$ is an identity).

It is an interesting exercise in categorical style constructions to show that this collection of data can be derived from a finite group $G$ acting on a normal variety $\tilde{X}$, plus open sets $\tilde{X}_{\alpha} \subset \tilde{X}$ stabilized by $H_{\alpha} \subset G$, very much as above:

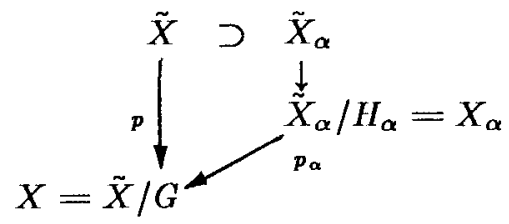

and satisfying:

a) For all $x \in \tilde{X}_{\alpha} \cap g\left(\tilde{X}_{\beta}\right)$ and all $h \in H_{\alpha}$ such that $h(x)=x$ then $g^{-1} h g \in H_{\beta}$.

b) $H_{\alpha}$ acts faithfully on $X_{\alpha}$.

Then define

$$
\begin{aligned}
X_{\alpha \beta}= & \coprod_{\substack{g=\text { represiof double } \\
\text { coseto } H_{\alpha} \backslash G / H_{\beta}}} \tilde{X}_{\alpha} \cap g\left(\tilde{X}_{\beta}\right) / H_{\alpha} \cap g H_{\beta} g^{-1} \\
p_{1}= & \text { natural map } \tilde{X}_{\alpha} \cap g\left(\tilde{X}_{\beta}\right) / H_{\alpha} \cap g H_{\beta} g^{-1} \rightarrow \tilde{X}_{\alpha} / H_{\alpha} \\
p_{2}= & \text { the map induced by } g^{-1} \\
& \tilde{X}_{\alpha} \cap g\left(\tilde{X}_{\beta}\right) / H_{\alpha} \cap g H_{\beta} g^{-1} \rightarrow \tilde{X}_{\beta} / H_{\beta}
\end{aligned}
$$

and if

$$
\begin{aligned}
& x \in \tilde{X}_{\alpha} \cap g\left(\tilde{X}_{\beta}\right) \text { maps to } \bar{x} \in X_{\alpha \beta} \\
& y \in \tilde{X}_{\boldsymbol{\beta}} \cap g^{\prime}\left(\tilde{X}_{\gamma}\right) \text { maps to } \bar{y} \in X_{\beta \gamma}
\end{aligned}
$$

${ }^{2}$ The maps o can also be introduced by giving as extra data more of a semi-simplicial variety:

in the usual way.

$$
\coprod x_{\alpha \beta \gamma} \equiv \coprod_{\alpha \beta}
$$


so that

$$
g^{-1} x=h y, \quad h \in H_{\beta}
$$

then let

$$
\left.\bar{x} \circ \bar{y}=\text { (image of } x \in \tilde{X}_{\alpha} \cap g h g^{\prime}\left(\tilde{X}_{\gamma}\right) \text { in } X_{\alpha \gamma}\right) \text {. }
$$

The object so constructed is a $Q$-variety if $G$ acts faithfully on $\tilde{X}$, but in general only a $Q$-stack.

Note that $\bar{M}_{2}$ and $\bar{M}_{1,1}$ are in a natural way $Q$-stacks. We let $\tilde{M}_{2}, \tilde{M}_{1,1}$ be the normalization of $\bar{M}_{2}, \bar{M}_{1,1}$ in the level $n$ covering, some $n \geq 3$ and let $G=S p(4, \mathbb{Z} / n \mathbb{Z}), S L(2, \mathbb{Z} / n \mathbb{Z})$ resp. The open sets $X_{\alpha}$ and subgroups $H_{\alpha}$ are defined exactly as in the case $g \geq 3$ treated above. The most general chart is any $X_{\alpha} \rightarrow \bar{M}_{2}$ (resp. $\bar{M}_{1,1}$ ) such that $X_{\alpha}$ comes with a family of the corresponding curves over it which represents locally everywhere the universal deformation space. Given 2 charts $X_{\alpha}, X_{\beta}$, with families $C_{\alpha}, C_{\beta}$, then $X_{\alpha \beta}$ is by definition:

$$
\operatorname{Isom}\left(C_{\alpha}, C_{\beta}\right)=\left\{(x, y, \phi) \mid x \in X_{\alpha}, y \in X_{\beta}, \phi \text { an isom. of } C_{\alpha, x} \text { with } C_{\beta, y}\right\}
$$

Firally morphisms between $Q$-stacks $X, Y$ are given by sets of morphisms and commuting diagrams:

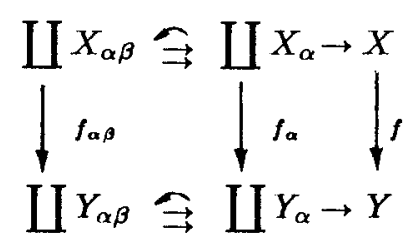

provided the atlas for $X$ is suitably refined. For suitable $\tilde{X}$ and $\tilde{Y}$ the morphism will be induced by a morhism

$$
\tilde{f}: \tilde{X} \rightarrow \tilde{Y}
$$

which is equivariant with respect to a homomorphism $G_{X} \rightarrow G_{Y}$ of the finite groups acting on $\tilde{X}, \tilde{Y}$. However, if the $Q$-slack $X$ is already presented as $\tilde{X} / G_{X}$ for one $\tilde{X}$, one may have to pass to a bigger covering before $\tilde{f}$ will be defined. This gives a diagram

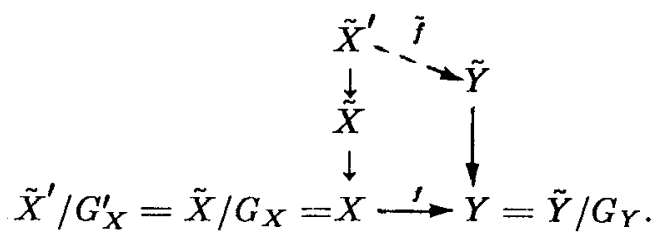


Among morphisms of $Q$-stacks, the simplest class consists of those that satisfy:

$$
\mid \begin{aligned}
& \forall \alpha, \text { let } X_{\alpha \alpha}^{\prime}=\left\{x \in X_{\alpha \alpha} \mid f_{\alpha \alpha}(x)=c_{Y}\left(f_{\alpha}\left(p_{1}(x)\right)\right)\right\} \\
& \text { Then } X_{\alpha \alpha}^{\prime} \text { acts freely on } X_{\alpha}
\end{aligned}
$$

These are the morphisms whose fibres are bona fide varieties, not just $Q$-varieties or $Q$-stacks. For such morphisms, it is possible to choose $\tilde{X}, \tilde{Y}$ with the same finite group $G$ acting and $\tilde{f} G$-equivariant:

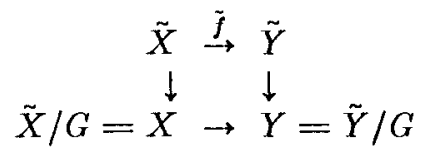

such that, moreover, locally, $\tilde{X}_{\alpha} \cong\left(\tilde{X}_{\alpha} / H_{\alpha}\right) \times_{\left(\tilde{Y}_{\alpha} / H_{\alpha}\right)} \tilde{Y}_{\alpha}$. The fibres of $\tilde{f}$ are then the "true fibres" of $f$. The typical example of this is

$$
\begin{array}{cc}
\bar{C}_{g}^{(n)} & \rightarrow \bar{M}_{g}^{(n)} \\
\downarrow & \downarrow \\
\bar{C}_{g} & \rightarrow \bar{M}_{g}
\end{array}
$$

where

$$
\begin{aligned}
\bar{C}_{g}= & \text { moduli space of pairs }(C, x), C \text { a stable } \\
& \text { curve, } x \in C \\
\cong & \text { moduli space of pairs }(C, x), \text { a 1-pointed } \\
& \text { stable curve }{ }^{3} \\
\bar{C}_{g}^{(n)}= & \text { normalization of } \bar{C}_{g} \text { in the covering } \\
& \text { defined by the inoduli space of triples } \\
& (C, x, \phi), C \text { a smooth curve, } x \in C \\
& \text { and } \phi \text { a level } n \text { structure on } C .
\end{aligned}
$$

Such morphisms may be called representable morphisms of $Q$-stacks. We do not need to develop the theory of $Q$-stacks for our applications, so we stop at merely these definitions.

${ }^{3}$ An $n$-pointed stable curve $\left(C, x_{1}, \ldots, x_{n}\right)$ is a reduced, connected curve $C$ with at most ordinary double points plus $n$ distinct smooth points $x_{i} \in C$ such that every smooth rational component $E$ of $C$ contains at least 3 points which are either $x_{i}$ 's or double points of $C$. 
§3. The Chow Group for $Q$-Varieties with Cohen-Macaulay Global Covers

We want to study a quasi-projective $Q$-variety $\left\{p_{\alpha}: X_{\alpha} \rightarrow X\right\}$ of dimension $n$ such that the big global cover $\tilde{X}$ is Cohen-Macaulay. We use the notation of $\S 2$, esp. $X=\tilde{X} / G$. We also choose a resolution of singularities

$$
\pi: \tilde{X}^{*} \rightarrow \tilde{X}
$$

Then we assert:

Theorem 3.1. With the above hypotheses, there is a canonical isomorphism $\gamma$ between the Chow group of $X$ and $G$-invariants in the operational Chow ring of $\tilde{X}$, (as usual after extending the coefficients to $\mathbb{Q}$ ):

$$
\gamma: A_{n-k}(X) \cong{ }^{k} A^{k}(\tilde{X})^{G}, \quad 0 \leq k \leq n .
$$

This key result does two important things for us:

a) it defines a ring structure on $A .(X)$,

b) for all $Q$-shcaves $\mathcal{F}$ on the $Q$-variety $X$, we can define Chern classes

$$
c_{k}(\mathcal{F}) \in A .(X) .
$$

I don't know if these things can be done if we drop the hypothesis that $\tilde{X}$ is Cohen-Macaulay. My guess is that this hypothesis can be dropped, but more powerful tools seem to be needed to treat this case.

Proof. The first step is to define, for all subvarieties $Z \subset X$, an element $\gamma(Z) \in o p A^{\cdot}(\tilde{X})^{C} \otimes \mathbb{Q}$. We use the local covers $p_{\alpha}: X_{\alpha} \rightarrow X$ and let $p_{\alpha}^{-1}(Z)$ be the reduced subscheme of $X_{\alpha}$ with support $p_{\alpha}^{-1}(Z)$. Then $\left\{O_{p_{\alpha}^{-1}(Z)}\right\}$ is a $Q$-sheaf on the $Q$-variety $X$. As above, let $p$ factor locally

$$
\tilde{X}_{\alpha} \stackrel{q_{\alpha}}{\rightarrow} \tilde{X}_{\alpha} / H_{\alpha} \cong X_{\alpha} \stackrel{p_{\alpha}}{\rightarrow} X
$$

and lift $p_{\alpha}^{-1}(Z)$ to its scheme-theoretic inverse image $q_{\alpha}^{*}\left(p_{\alpha}^{-1}(Z)\right)$. Define

$$
\tilde{Z}=\left\{\begin{array}{l}
G \text {-invariant subscheme of } \tilde{X} \text { supported } \\
\text { on } p^{-1}(Z) \text { such that }\left.\tilde{Z}\right|_{\tilde{X}_{\alpha}}=q_{\alpha}^{*}\left(p_{\alpha}^{-1}(Z)\right)
\end{array}\right\} .
$$


Note that because $\tilde{X}$ is Cohen-Macaulay, and $X_{\alpha}$ is smooth, $q_{\alpha}$ is flat. Since $O_{p_{\alpha}^{-1}(Z)}$ has a finite projective resolution on $X_{\alpha}$, this implies that $O_{Z}$ has a finite projective resolution on $\tilde{X}$. Therefore, by Fulton's theory $[\mathrm{F} 1]$, the Chern classes $c_{k}\left(O_{\tilde{Z}}\right)$ are defined in $o p A^{k}(\tilde{X})$. Next define the "ramification index":

$$
e(Z)=\text { order of the stabilizer in } G_{\alpha} \text { of almost all points of } p_{\alpha}^{-1}(Z) .
$$

Here $\alpha$ is any index such that $p_{\alpha}^{-1}(Z) \neq \phi$ : the definition does not depend on $\alpha$. Then let

$$
\begin{aligned}
& \gamma(Z)=\frac{(-1)^{k-1} e(Z)}{(k-1) !} \cdot c_{k}\left(O_{\tilde{Z}}\right), \quad \text { if } k=\operatorname{codim} Z \\
& \left(\text { or }=e(Z) c h_{k}\left(O_{\tilde{Z}}\right), \text { using lemma } 3.3\right. \text { below) }
\end{aligned}
$$

An important point in the study of $\tilde{Z}$ is that the family of subschemes $\left\{p_{\alpha}^{-1}(Z)\right\}$ can be simultaneously resolved:

Theorem 3.2 (Hironaka). For all subvarieties $Z \subset X$, there is a birational map $\pi: Z^{*} \longrightarrow Z, Z^{*}$ normal, such that for all $\alpha$

$$
\left(p_{\alpha}^{-1}(Z) \times{ }_{Z} Z^{*}\right)_{n o r}
$$

is smooth.

This is a Corollary of Hironaka's strong resolution theorem, giving a resolution compatible with the pseudo-group of all local analytic isomorphisms between open sets in the original variety: see [H], Theorem 7.1, p. 164. One may proceed as follows: first resolve $p_{\alpha_{1}}^{-1}(Z)$ as in $[\mathrm{H}]$. Since $\left(\left(\tilde{X}_{\alpha_{1}} / H_{\alpha}\right) \times_{X}\left(\tilde{X}_{\alpha_{1}} / I_{\alpha_{1}}\right)\right)_{n o r}$ is étale over $\tilde{X}_{\alpha_{1}} / H_{\alpha_{1}}$ this equivalence relation extends to one on the resolution $p_{\alpha_{1}}^{-1}(Z)^{*}$, hence there is a blow-up $\pi_{1}: Z_{1} \longrightarrow Z$ such that $p_{\alpha_{1}}^{-1}(Z)^{*} \cong\left(p_{\alpha_{1}}^{-1}(Z) \times_{Z} Z_{1}\right)_{\text {nor }}$. Secondly, resolve $\left(p_{\alpha_{2}}^{-1}(Z) \times{ }_{Z} Z_{1}\right)_{n o r}$ by a blow-up over $X-p\left(X_{\alpha_{1}}\right)$ so as not to affect the first step. Again, descend this to a further blow-up $\left(\pi_{2}: Z_{2} \rightarrow Z_{1} \rightarrow Z\right)$ of $Z$. Eventually, we get the needed resolution.

Lemma 3.3. For all $Q$-sheaves $\mathcal{F}$ on the $Q$-variety $X$, let $S \subset X$ be the support of $\mathcal{F}$. Then in opA $(\tilde{X})$, 
a) $c_{k}(\tilde{f})=0$ if $k<\operatorname{codim} S$

b) if $k=\operatorname{codim} S$, and $S_{1}, \ldots, S_{n}$ are the codimension $k$ components of $S$, then

$$
c_{k}(\tilde{\mathcal{F}})=\sum_{i=1}^{n} l_{i} c_{k}\left(O_{\tilde{S}_{i}}\right)
$$

where $l_{i}$ is the length of the stalk of $\mathcal{F}_{\alpha}$ at the generic point of $p_{\alpha}^{-1}\left(S_{i}\right)$ when $S_{i} \operatorname{mets} p\left(\tilde{X}_{\alpha}\right)$.

Proof. This results from an application of Fulton's Grothendieck-Riemann-Roch Theorem 1.4 and Hironaka's resolution 3.2. By the usual dévissage, reduce the lemma to the case where $S$ is irreducible of codimension $k$, and $\tilde{\mathcal{F}}$ is an $O_{\tilde{S}}$-module. Let $S^{*} \rightarrow S$ be a "resolution" as in 3.2 . This gives a family of resolutions

$$
S_{\alpha}^{*}=\left(p_{\alpha}^{-1}(S) \times{ }_{S} S^{*}\right)_{n o r} \stackrel{\pi_{\alpha}}{\longrightarrow} p_{\alpha}^{-1}(S) \subset \tilde{X}_{\alpha} / H_{\alpha}
$$

which are local complete intersection (or l.c.i.) morphisms. Therefore, by fibre product with the flat morphism $\tilde{X}_{\alpha} \rightarrow \tilde{X}_{\alpha} / H_{\alpha}$,

$$
S_{\alpha}^{*} \times_{\left(\tilde{X}_{\alpha} / H_{\alpha}\right)} \tilde{X}_{\alpha} \rightarrow \tilde{X}_{\alpha}
$$

are l.c.i. morphisms. These glue together to an l.c.i. morphism

$$
\tilde{S}^{*} \stackrel{\pi}{\rightarrow} \tilde{X}
$$

such that $\tilde{\mathcal{F}}$ is a $\pi_{*} O_{\tilde{S}}{ }^{*-\bmod u l e . ~ L e t ~} \tilde{\mathcal{F}}^{*}=\pi^{*}(\tilde{\mathcal{F}})$. Then by 1.4 :

$$
\operatorname{ch} \pi_{!}\left(\tilde{\mathcal{F}}^{*}\right)=\pi_{*}\left(\operatorname{ch} \tilde{\mathcal{F}}^{*} \cdot T d_{\pi}\right) .
$$

Now if $i>0, R^{i} \pi_{*}\left(\tilde{f}^{*}\right)$ are $Q$-sheaves on the $Q$-variety $X$ with supports properly contained in $S$, so by induction we can assume

$$
c_{l}\left(R^{i} \pi_{*} \tilde{\mathcal{F}}^{*}\right)=0 \quad \text { if } \quad l \leq k, i>0 .
$$

Therefore

$$
\operatorname{ch}\left(\pi_{1} \tilde{\mathcal{F}}^{*}\right)=\operatorname{ch}_{l}\left(\pi_{*} \tilde{\mathcal{F}}^{*}\right)=\operatorname{ch}_{l}(\tilde{\mathcal{F}}) \quad \text { if } \quad l \leq k .
$$

But $\pi_{*}: o p \Lambda\left(\tilde{S}^{*}\right) \rightarrow o p \Lambda(\tilde{X})$ raises the codimension of a cycle by $k$. So

$$
c h_{l}(\tilde{F})=0 \quad \text { if } \quad l<k
$$


and

$$
\begin{aligned}
& c h_{k}(\tilde{F})=\left(\text { generic rank of } \tilde{\mathcal{F}}^{*} \text { as free } O_{\tilde{S}^{*-\operatorname{modul}}}\right) \cdot \pi_{*}(1) \\
& =\left(\text { length of } f_{\alpha} \text { at generic point of } p_{\alpha}^{-1}(S)\right) \cdot c h_{k}\left(O_{\tilde{S}}\right) \text {. }
\end{aligned}
$$

Q.E.D.

Lemma 3.4. If two cycles $\sum n_{i} Z_{i}, \sum m_{i} W_{i}$ on $X$ are rationally equivalent, then

$$
\sum n_{i} \gamma\left(Z_{i}\right)=\sum m_{i} \gamma\left(W_{i}\right) \quad \text { in op } A(\tilde{X})
$$

Proof. Let $L$ be an ample line bundle on $X$. Rational equivalence on $X$ may be defined by requiring that for all subvarieties $Y \subset X$ and all $s_{1}, s_{2} \in \Gamma\left(Y, L^{n} \otimes O_{Y}\right)$, if $D_{i}$ is the divisor of zeroes of $s_{i}$ on $X$, then

$$
D_{1} \underset{r a t}{ } D_{2} \text {. }
$$

So to prove the lemina, it will suffice to prove that for all $s \in \Gamma\left(Y, L^{n} \otimes O_{Y}\right)$, if $D=\sum n_{i} Z_{i}$ is the divisor of zeroes of $s$, then

$$
\sum n_{i} \gamma\left(Z_{i}\right)=e(Y) \cdot\left[c h_{k}\left(O_{\bar{Y}}\right)-c h_{k}\left(L^{-n} \otimes O_{Y}\right)\right]
$$

To see this, use the cxact sequence of $Q$-sheaves

$$
0 \rightarrow L^{-n} \otimes O_{\tilde{Y}} \stackrel{\otimes s}{\rightarrow} O_{\tilde{Y}} \rightarrow O_{\tilde{D}} \rightarrow \mathbf{0}
$$

where $\tilde{D} \subset \tilde{X}$ is the scheme of zeroes of $s$ in $\tilde{Y}$ and the local calculation ${ }^{4}$ that on $p^{-1}(Y)$ :

(*) (Divisor of zeroes of $p_{\alpha}^{*}(s)$ on $\left.p_{\epsilon}^{-1}(Y)\right)=\sum n_{i} \frac{e\left(Z_{i}\right)}{e(Y)} p_{\alpha}^{-1}\left(W_{i}\right)$

\footnotetext{
${ }^{4}$ We use the lemma that if a finite group $G$ acts faithfully on a variety $Y$ and $\phi$ is a $G$-invariant function on $Y$, zcro on a subvariety $W \subset Y$ of codimension 1 , and $\bar{\phi}$ is the induced function on $Y / G$, then:

$$
\operatorname{ord}_{W}(\phi)=\#\{g \in G \mid g=i d . \text { on } W\} \cdot \operatorname{ord}_{W / G}(\bar{\phi}) \text {. }
$$
}


(n.b., $p_{\alpha}^{-1}(Y), p_{\alpha}^{-1}\left(W_{i}\right)$ are the reduced inverse images). Therefore

$$
\begin{aligned}
c h_{k} O_{\tilde{Y}}-c h_{k} L^{-n} \otimes O_{\tilde{Y}} & =c h_{k} O_{\tilde{D}} \\
& =\frac{(-1)^{k-1}}{(k-1) !} c_{k} O_{\tilde{D}} \\
& =\sum_{i}\left(\begin{array}{l}
\text { length of } O_{D_{\alpha}} \text { at } \\
\text { gen.pt. of } p_{\alpha}^{-1}\left(W_{i}\right)
\end{array}\right) \cdot \frac{(-1)^{k-1}}{(k-1) !} c_{k} O_{\tilde{W}_{i}} \\
& =\sum_{i} \text { ord }_{p_{\alpha}^{-1}\left(W_{i}\right)}\left(p_{\alpha}^{*} s\right) \cdot \frac{\gamma\left(W_{i}\right)}{e\left(W_{i}\right)} \quad \text { by def }{ }^{\underline{n}} \text { of } \gamma \\
& =\frac{1}{e(Y)} \sum n_{i} \gamma\left(W_{i}\right) \quad \text { by }(*) .
\end{aligned}
$$

This proves (3.4), which shows that $\gamma$ factors:

$$
\gamma: A .(X) \rightarrow o p A^{\prime}(\tilde{X})^{G} .
$$

Lemma 3.5. The composition of maps

$$
A .(X) \stackrel{\gamma}{\longrightarrow} o p A^{*}(\tilde{X})^{G \stackrel{\cap[\tilde{X}]}{\longrightarrow}} A .(\tilde{X})^{G \stackrel{p *}{\longrightarrow}} A .(X)
$$

is multiplication by $n$, the degree of $p$.

Proof. To prove this we use another Ricmann-Roch theorem: the version of Baum-Fulton-MacPherson [BFM]. This says that there is a natural transformation $\tau: K_{o}(Z) \rightarrow A .(Z)$ for all varicties $Z$ such that

$$
\begin{aligned}
& K^{\circ}(Z) \otimes K_{o}(Z) \stackrel{\otimes}{\rightarrow} K_{o}(Z) \\
& \downarrow c h \otimes r \quad \downarrow r \\
& o p \Lambda \cdot(Z) \otimes A .(Z) \stackrel{\cap}{\rightarrow} \Lambda .(Z)
\end{aligned}
$$

commutes. By the lemma, p. 129 of $[\mathrm{BFM}]$ and dévissage, $\tau$ satisfies:

for all $\mathcal{F}$ with support $\cup Z_{i}$ of codimension $k, \tau(\mathcal{F})$ has codimension $k$ and

$$
\tau(\mathcal{F})_{k}=\text { class of } \sum\left(\begin{array}{l}
\text { length of } \mathcal{F} \text { at } \\
\text { gen. pt. of } Z_{i}
\end{array}\right)\left[Z_{i}\right] .
$$


We apply this to $Z=\tilde{X}$ and $\mathcal{I}=\mathcal{O}_{\tilde{Z}}$ where $Z \subset X$ is a subvariety. It follows that

$$
\operatorname{ch}\left(O_{\tilde{Z}}\right) \cap \tau\left(O_{\tilde{X}}\right)=\tau\left(O_{\check{Z}}\right)
$$

Therefore if $k=$ codimension $Z$,

$$
\begin{aligned}
p_{*}(\gamma(Z) \cap[\tilde{X}]) & =e(Z) \cdot p_{*}\left(\operatorname{ch}_{k}\left(O_{\tilde{Z}}\right) \cap[\tilde{X}]\right) \\
& =e(Z) \cdot p_{*}([\tilde{Z}]) \\
& =e(Z) \cdot[\tilde{Z}: Z] \cdot \text { class of } Z \\
& =n \cdot \text { class of } Z
\end{aligned}
$$

Q.E.D.

Lemma 3.6. If $\pi: \tilde{X}^{*} \rightarrow \tilde{X}$ is a resolution, and $Z \subset X$ is a subvariety of codimension $k$ such that for all components $Z_{i}$ of $p^{-1}(Z), \pi^{-1}\left(Z_{i}\right)$ is irreducible of codimension $k$, then $\pi^{*}(\gamma(Z))$ is represented by a cycle

$$
c \cdot \sum \pi^{-1}\left(Z_{i}\right), \quad \text { some } c \in \mathbb{Q}, c>0 \text {. }
$$

Proof. Let $U \subset \tilde{X}$ be the open set over which $\pi$ is an isomorphism. Then $Z_{i} \cap U \neq \phi$, all $i$. Now

$$
\begin{aligned}
\pi^{*}(\gamma(Z)) & =e(Z) \cdot \pi^{*}\left(\operatorname{ch}_{k}\left(O_{\tilde{Z}}\right)\right) \\
& =e(Z) \cdot\left(\sum(-1)^{l} \operatorname{ch}_{k}\left(\operatorname{tor}_{l}\left(O_{\tilde{Z}}, O_{\tilde{X}^{*}}\right)\right)\right)
\end{aligned}
$$

But these tor $l$ are supported on proper subsets of $\pi^{-1}\left(Z_{i}\right)$, hence have no $k^{\text {th }}$ Chern character. Therefore:

$$
\begin{aligned}
\pi^{*}(\gamma(Z)) & =e(Z) \cdot \operatorname{ch}_{k}\left(O_{\tilde{Z}} \otimes O_{\tilde{X}^{*}}\right) \\
& =e(Z) \cdot \text { class of } \pi^{-1}(\tilde{Z})
\end{aligned}
$$

by the Riemann-Roch theorem on $\tilde{X}^{*}$.

Q.E.D.

Corollary 3.7. $\quad \gamma$ is bijective.

Proof. $3.5,3.6$ and 1.2 . 
This proves the theorem. $\Lambda$ few comments can be made on the ring structure that this introduces in $A .(X)$. First of all, suppose $W_{1}, W_{2}$ are two cycles on $X$ that intersect properly. Then the product $\left[W_{1}\right],\left\{W_{2}\right]$ in the above ring structure can also be defined directly by assigning suitable multiplicities to the components of $\operatorname{Supp} W_{1} \cap \operatorname{Supp} W_{2}$. In fact, define:

$$
W_{1} \cdot W_{2}=\sum_{\substack{\text { compp.Uof } \\ \text { supp } W_{1} \cap \mathrm{Nupp} W_{2}}} i\left(W_{1} \cap W_{2} ; U\right) \cdot U
$$

where if $p_{\alpha}^{-1}(U) \neq \phi$, then

$$
i\left(W_{1} \cap W_{2} ; U\right)=\frac{e\left(W_{1}\right) \cdot e\left(W_{2}\right)}{e(U)} \cdot i\left(p_{\alpha}^{-1}\left(W_{1}\right) \cap p_{\alpha}^{-1}\left(W_{2}\right) ; p_{\alpha}^{-1} U\right) .
$$

Note that the intersection multiplicity on the right is taken on the smooth ambient variety $\tilde{X}_{\alpha} / H_{\alpha}$, hence is defined, e.g., by

$$
\sum_{l}(-1)^{l}\left(\begin{array}{l}
\text { length at gen. pt. } \\
\text { of } p_{\alpha}^{-1} U
\end{array}\right)\left(\operatorname{tor}_{l}\left(O_{p_{\alpha}^{-1} W_{1}}, O_{p_{\alpha}^{-1} W_{2}}\right)\right)
$$

The proof that this is the same as the product in $o p A^{\cdot}(\tilde{X})$ is straightforward, i.e.,

$$
\begin{aligned}
\gamma\left(Z_{1}\right) \cdot \gamma\left(Z_{2}\right)= & e\left(Z_{1}\right) e\left(Z_{2}\right) c h_{k_{1}}\left(O_{\tilde{Z}_{1}}\right) \cdot c h_{k_{2}}\left(O_{\tilde{Z}_{2}}\right) \\
= & e\left(Z_{1}\right) e\left(Z_{2}\right) c h_{k_{1}+k_{2}}\left(O_{\tilde{Z}_{1}} \stackrel{\mathrm{L}}{\otimes} O_{\tilde{Z}_{2}}\right) \\
& (\stackrel{\mathrm{L}}{\otimes} \text { means take tensor product of projective resol. }) \\
= & \sum_{U} e\left(Z_{1}\right) e\left(Z_{2}\right) i\left(p_{\alpha}^{-1}\left(W_{1}\right) \cap p_{\alpha}^{-1}\left(W_{2}\right) ; p_{\alpha}^{-1} U\right) \cdot c h_{k_{1}+k_{2}}\left(O_{\tilde{U}}\right) \\
= & \sum \frac{e\left(Z_{1}\right) e\left(Z_{2}\right)}{e(U)} i\left(p_{\alpha}^{-1}\left(W_{1}\right) \cap p_{\alpha}^{-1}\left(W_{2}\right) ; p_{\alpha}^{-1} U\right) \cdot \gamma(U) .
\end{aligned}
$$

This product could be introduced directly without relating it to the product in $o p A^{\prime}(\tilde{X})$. This has been done by Matsusaka in his book "Theory of $Q$-varieties", [Ma], where associativity and other standard formulae are proven. The missing ingredient, however, is the moving lemma. This follows as a Corollary of the isomorphism of $A .(X)$ with $o p A \cdot(\tilde{X})$, i.e., by representing a cycle on $X$ as the projection from $X$ of the Chern class of 
a sheaf with finite resolution. In particular, I don't know any way to get a moving lemma unless some $\tilde{X}$ is Cohen-Macaulay.

IIenceforth, in the study of the Chow rings of $Q$-varieties we shall identify $A_{n-k}(X)$ and $o p A^{k}(\tilde{X})^{G}$ via the map $\gamma$, and write this as $A^{k}(X)$ just like the $k$-codimension piece of the Chow ring of an ordinary non-singular variety. This does not usually lead to any confusion, except with regard to the concept of the fundamental class of a subvariety $Y \subset X$. The important thing to realize here is that there are really two different notions of fundamental class, differing by a rational number, and both are important. Thus for all $Y$ of codimension $k$, we will write

$$
\begin{aligned}
{[Y]=} & \text { class of the cycle } Y \text { in the Chow group } \\
& A_{n-k}(X)=A^{k}(X)
\end{aligned}
$$

and

$$
[Y]_{Q}=\text { the class } c h_{k}\left(O_{\tilde{Y}}\right) \text { in } o p A^{k}(\tilde{X})^{G}=A^{k}(X)
$$

Since we are using the identification $\gamma$, we have:

$$
[Y]_{Q}=\frac{1}{e(Y)} \cdot[Y]
$$

When one makes calculations of intersections in local charts $\tilde{X}_{\alpha} / H_{\alpha}$, then one is verifying an identity between classes $[Y]_{Q}$. But when one has a rational equivalence between cycles on $X$, one has an jdentity between $[Y]$ 's: c.g., if $X$ is unirational, then for all points $P_{1}, P_{2} \in X$,

$$
\left[P_{1}\right]=\left[P_{2}\right]
$$

but the point classes $[P]_{Q}$ are fractions $1 / e(P)$ of the basic point class $[P] \in A^{\prime}(X)$.

If $X$ is a $Q$-stack, exactly the same theorem holds and we have an isomorphism

$$
\gamma: A .(X) \approx o p A(\tilde{X})^{G} \text {. }
$$

The only difference is that a subgroup $Z \subset G$ acts identically on $\tilde{X}$. If $\# Z=z$, then the effect of this is merely to modify the ring structure on $A^{\cdot}(X)$ as follows. Let $W_{1}, W_{2}$ be cycles on $X$ and consider:

i) the $Q$-variety structure on $X$ given by the action of $G / Z$ on $\tilde{X}$, and the multiplication $W_{1 \cdot v a r} W_{2}$, 
ii) the $Q$-stack structure on $X$ given by the action of $G$ on $\tilde{X}$, and the inultiplication $W_{\mathrm{l} \cdot s t} W_{2}$.

Then by the moving lemma plus the formula above for proper intersections, it follows:

$$
W_{1 \cdot s t} W_{2}=z \cdot W_{1 \cdot v a r} W_{2}
$$

In particular, the identity in the Chow ring of a $Q$-stack $X$ is $[X]_{Q}$, $\operatorname{not}[X]$.

The Chow ring for $Q$-varieties, or more generally $Q$-stacks, has good contravariant functorial properties. We consider morphisms of $Q$-stacks with global Cohen-Macaulay covers:

$$
X \stackrel{f}{\rightarrow} Y
$$

as defined in $\S 2$. Then I clairn:

Proposition 3.8. There is a canonical ring homomorphism

$$
f^{*}: A(Y) \rightarrow A(X)
$$

satisfying:

i) $f_{*}\left(a . f^{*} b\right)=f_{*} a . b \quad\left(f_{*}\right.$ being defined from $A .(X)$ to $A .(Y)$ as usual $)$,

ii) $f^{*}\left(c_{k} \mathcal{E}\right)=c_{k}\left(f^{*} \mathcal{E}\right)$ for all $Q$-vector bundles $\mathcal{E}$ on $Y$

iii) if $W$ is a subvariety of $Y$ such that codim $f^{-1}(W)=\operatorname{codim} W$ then

$$
f^{*}([W])=\text { class of } \sum_{\substack{\text { comp. } V_{k} \\ \text { of } f^{-1}(w)}} i_{k} \cdot\left[V_{k}\right]
$$

where $i_{k}$ is calculated on suitable charts $f_{\alpha}: X_{\alpha} \rightarrow Y_{\alpha}$ by pull-backs in the smooth case adjusted by $e(W) / e\left(V_{k}\right)$.

Proof. Although the moving lemma plus (iii) provides us with the simplest formula for $f^{*}$, to see that $f^{*}$ is well-defined, we use op $A^{\text {. There is one }}$ complication. $X$ and $Y$ have global Cohen-Macaulay covers $\tilde{X}, \tilde{Y}$ but $f$ may 
not lift to $\tilde{f}: \tilde{X} \rightarrow \tilde{Y}$. Instead, we may have to 'refine' $\tilde{X}$ :

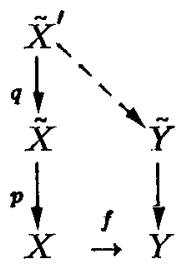

and then $\tilde{X}^{\prime}$ may not longer be Cohen-Macaulay. Still, once one has one Cohen-Macaulay $\tilde{X}$ with which to set up the theory, one proves that

$$
o p A^{\cdot}(X)^{G_{X} \quad a^{*}} \quad o p A^{\cdot}\left(\tilde{X}^{\prime}\right)^{G_{X}^{\prime}}
$$

is an isomorphism, hence $f^{*}$ may be defined by:

$$
A .(Y) \underset{\gamma_{Y}}{\approx} o p A^{\cdot}(\tilde{Y})^{G_{Y}} \underset{\tilde{j}_{.}}{\rightarrow} o p A^{\cdot}\left(\tilde{X}^{\prime}\right)^{G_{X}^{\prime}} \approx o p A^{\cdot}(\tilde{X})^{G_{X}} \approx A .(X) .
$$

'To check (3.9), use

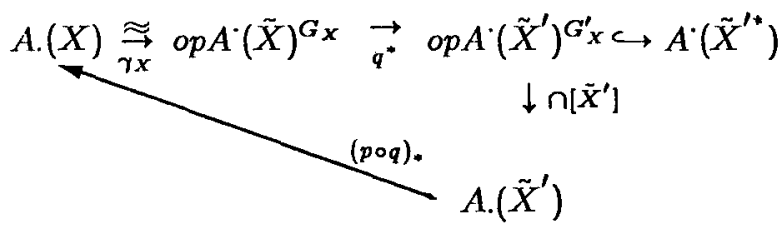

$\left(\tilde{X}^{\prime *}=\right.$ resolution of $\left.\tilde{X}^{\prime}\right)$ and argue as in lemmas 3.5 and 3.6. There is one hitch: namely, in 3.5 , we get

$$
\begin{aligned}
(p \circ q)_{*}\left(q^{*}(\gamma(Z)) \cap\left[\tilde{X}^{\prime}\right]\right) & =e(Z)(p \circ q)_{*}\left(q^{*}\left(\operatorname{ch}_{k}\left(O_{\tilde{Z}}\right)\right) \cap\left[\tilde{X}^{\prime}\right]\right) \\
& =e(Z)(p \circ q)_{*}\left(\operatorname{ch}_{k}\left(O_{\tilde{Z}} \otimes O_{\tilde{X}^{\prime}}\right) \cap\left[X^{\prime}\right]\right)
\end{aligned}
$$

where $\stackrel{\mathrm{L}}{\otimes}$ means take a resolution of $O_{\tilde{Z}}$ and tensor it with $O_{\tilde{X}^{\prime}}$. But now if $\tilde{X}^{\prime}$ is not Cohen-Macaulay, $O_{\tilde{Z}} \otimes O_{\tilde{X}^{\prime}}$ will not be a resolution of some $O_{\tilde{Z}^{\prime}}$ and we get instead:

$$
\begin{aligned}
& =e(Z) \sum(-1)^{l}(p \circ q)_{*}\left(c h_{k} \operatorname{tor}_{l}\left(O_{\tilde{Z}}, O_{X^{\prime}}\right) \cap\left[\tilde{X}^{\prime}\right]\right) \\
& =e(Z) \cdot(p \circ q)_{*}\left(\sum_{n} i_{n} \cdot\left[\tilde{Z}_{n}\right]\right)
\end{aligned}
$$


where $\tilde{Z}_{l}$ are the components of $(p \circ q)^{-1} Z$ and

$$
\begin{aligned}
i_{n} & =\sum(-1)^{l}\left(\text { length at gen. pt. of } \tilde{Z}_{n}\right)\left(\operatorname{tor}_{l}^{o_{\tilde{X}}}\left(O_{\tilde{Z}}, O_{\tilde{X}^{\prime}}\right)\right) \\
& =\sum(-1)^{l}\left(\text { length at gen. pt. of } \tilde{Z}_{n}\right)\left(\operatorname{tor}_{l}^{o_{X_{\alpha}}}\left(O_{p_{\alpha}^{-1}(Z)}, O_{\tilde{X}^{\prime}}\right)\right) \\
& =\text { mult. of } \tilde{Z}_{n} \text { in the cycle } q_{\alpha}^{\prime *}\left(p_{\alpha}^{-1}(Z)\right)
\end{aligned}
$$

where we factor $p \circ q$ :

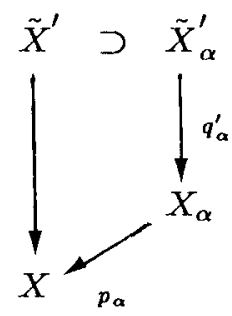

Thus

$$
\begin{aligned}
e(Z)(p \circ q)_{*}\left(\sum_{n} i_{n}\left[\tilde{Z}_{n}\right]\right) \\
=e(Z) p_{\alpha, *}\left(\text { class of } q_{\alpha, *}^{\prime}\left(q_{\alpha}^{\prime *}\left(p_{\alpha}^{-1} Z\right)\right)\right) \\
=\operatorname{deg} q_{\alpha}^{\prime} \cdot e(Z) \cdot p_{\alpha, *}\left(p_{\alpha}^{-1} Z\right) \\
=n . \operatorname{class} \text { of } Z .
\end{aligned}
$$

$f^{*}$ being defined, the rest of the proof is straightforward.

For representable morphisms $f: X \rightarrow Y$ of $Q$-stacks, there is a further important compatibility. For such $f$, let

$$
\tilde{f}: \tilde{X} \longrightarrow \tilde{Y}
$$

be a $G$-equivariant morphism such that $X=\tilde{X} / G, Y=\tilde{Y} / G$ and

$$
\tilde{X}_{\alpha} \cong X_{\alpha} X_{Y_{\alpha}} \tilde{Y}_{\alpha}
$$

as in $\S 2 b$. Then we have:

Proposition 3.10. If the morphism $f$ on local charts

$$
f_{\alpha}: X_{\alpha} \rightarrow Y_{\alpha}
$$


is a local complete intersection and $\tilde{Y}$ is Cohen-Macaulay, then $\tilde{f}$ is l.c.i. and the diagram

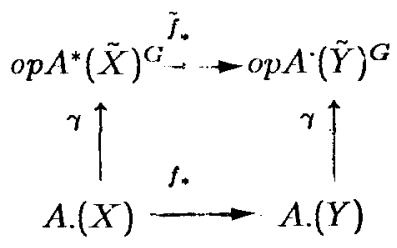

cornmutes.

Proof. Let $W$ be a codimension $k$ subvarinty of $X$ such that $W$ is generically finite over $f(W)$. Then we must check

$$
\tilde{f}_{*}(\gamma W)=[W: f W] \cdot \gamma(f W)
$$

i.e.,

$$
e(X) \cdot \tilde{f}_{*}\left(c h_{k}\left(O_{\tilde{W}}\right)\right)=[W: f W] \cdot e(f W) \cdot c h_{k}\left(O_{\delta} \tilde{W}\right)
$$

But by Riemann-Roch for $\tilde{f}$,

$$
\begin{aligned}
\tilde{f}_{*}\left(\operatorname{ch}_{k}\left(O_{\tilde{W}}\right)\right) & =c h_{k}\left(\tilde{f}_{*} O_{\tilde{W}}\right) \\
& =\left[W_{\alpha}: f_{\alpha}\left(W_{\alpha}\right)\right] \cdot \operatorname{ch} h_{k}\left(O_{f \tilde{W}}\right)
\end{aligned}
$$

because $\tilde{f}_{*}\left(O_{\tilde{W}}\right)$ is generically a locally free $O_{f} \tilde{W}^{\text {-algebra }}$ of length $\left[W_{\alpha}: f_{\alpha} W_{\alpha}\right]$ by (3.9). But now use:

$$
\left[W_{\alpha}: W\right] .[W: f W]=\left[W_{\alpha}: f_{\alpha} W_{\alpha}\right] \cdot\left[\int_{\alpha} W_{\alpha}: f W\right]
$$

and

$$
\begin{aligned}
{\left[W_{\alpha}: W\right] \cdot e(W) } & =\left[X_{\alpha}: X\right] \cdot e(X) \\
& =\left[G: H_{\alpha}\right] \\
& =\left[Y_{\alpha}: Y\right] \cdot e(Y) \\
& =\left[f(W)_{\alpha}: f W\right] \cdot e(f W)
\end{aligned}
$$

and the equality of the coefficients follows. 


\section{Part II: Basic Classes in the Chow Ring of the Moduli Space}

\section{§4. Tautological Classes}

Whenever a variety or topological space is defined by some universal property, one expects that by virtue of its defining property, it possesses certain cohomology classes called tautological classes. The standard example is a Grassmannian, e.g., the Grassmannian Grass of $k$-planes in $\mathbb{C}^{n}$. By its very definition, there is a universal bundle $E$ on Grass of rank $k$, and this induces Chern classes $c_{l}(E), 1 \leq l \leq k$, in both the cohomology ring of Grass and the Chow ring of Grass. These two rings are, in fact, isomorphic and generated as rings by $\left\{c_{l}(E)\right\}$. Moreover, one gets tautological relations from the fact that $E$ is a sub-bundle of the trivial bundle $\mathbb{C}^{n} \times$ Grass. This gives an exact sequence:

$$
0 \rightarrow E \rightarrow \mathrm{O}^{n} \rightarrow F \rightarrow 0, \quad F \text { a bundle of rank } n-k
$$

hence

$$
\left(1+c_{1}(E)+\cdots+c_{k}\left(E^{\prime}\right)\right)_{l}^{-1}=0, \quad l>n-k .
$$

As is well known, these are a complete set of relations for the cohomology and Chow rings of Grass.

We shall begin a program of the same sort for the Chow ring (or cohomology ring) of $\bar{M}_{g}$. Our purpose is merely to identify a natural set of tautological classes and some tautological relations. To what extent these lead to a presentation of either ring is totally unclear at the moment.

The natural place to start is with the universal curve over $\overline{\mathcal{M}}_{g}$. This is the same as the coarse moduli space of 1-pointed stable curves $(C, P)$ (see Knudsen $[\mathrm{K}]$, Harris-Mumford $[\mathrm{H}-\mathrm{M}]$ ), which we call $\bar{M}_{g, 1}$ or $\bar{C}_{g}$ alternatively. $\bar{C}_{g}$ is a $Q$-variety, too, and everything we have said about $\bar{M}_{g}$ applies to $\bar{C}_{g}$ too. The morphism $\bar{C}_{g} \rightarrow \bar{M}_{g}$ is a representable morphism, and via level $n$ structures, we have a covering $\tilde{C}_{g}$ of $\bar{C}_{g}$ and a morphism:

$$
\pi: \tilde{C}_{g} \longrightarrow \tilde{\mathcal{M}}_{g}
$$

which is a flat, proper family of stable curves, with a finite group $G$ acting on both, and $\bar{C}_{g}=\tilde{C}_{g} / G, \overline{\mathcal{M}}_{g}=\tilde{\mathcal{M}}_{g} / G$. If $g \geq 3$, then

$$
\tilde{C}_{g}=\text { (normalization of } \bar{C}_{g} \times_{\overline{\mathcal{M}}_{\mathrm{g}}} \tilde{\mathcal{M}}_{g} \text { ), }
$$


but if $g=2$, the generic curve has automorphisms, $S p(4, \mathbb{Z} / n \mathbb{Z})$ does not act faithfully on $\tilde{M}_{2}$, and $\tilde{C}_{2}$ is a double cover of this normalization. In any case, $\bar{C}_{g}$ has a $Q$-sheaf $\omega_{\bar{C}_{g} / \bar{M}_{0}}$ represented by the invertible sheaf $\omega_{\tilde{C}_{g}} / \overline{\mathcal{M}}_{g}$ on $\tilde{C}_{g}$. Henceforth, whenever we talk of sheaves on $\bar{C}_{g}$ or $\bar{M}_{g}$ we shall mean $Q$-sheaves and they are always represented by usual coherent sheaves on $\tilde{C}_{g}$ and $\tilde{M}_{g}$ with $G$-action. Furthermore, we shall make calculations in $A^{\cdot}\left(\bar{M}_{g}\right)$ and $A \cdot\left(\bar{C}_{g}\right)$ by implicitly identifying these with $o p A\left(\tilde{\mathcal{M}}_{g}\right)^{G}$ and $o p A \cdot\left(\tilde{C}_{g}\right)^{G}$.

Now define the tautological classes:

$$
\begin{aligned}
K_{\overline{\mathcal{C}}_{g} / \overline{\mathcal{M}}_{g}} & =c_{1}\left(\omega_{\overline{\mathcal{C}}_{g} / \overline{\mathcal{M}}_{g}}\right) \in A^{1}\left(\overline{\mathcal{C}}_{g}\right) \\
\kappa_{l} & =\left(\pi_{*} K_{\overline{\mathcal{C}}_{g} / \overline{\mathcal{M}}_{g}}\right) \in A^{l}\left(\overline{\mathcal{M}}_{g}\right) \\
\mathbb{E} & =\pi_{*}\left(\omega_{\overline{\mathcal{M}}_{a} / \overline{\mathcal{M}}_{g}}\right): \text { a locally free } Q \text {-sheaf of rank } g \text { on } \overline{\mathcal{M}}_{g} \\
\lambda_{l} & =c_{l}(\mathbb{E}), \quad 1 \leq l \leq g .
\end{aligned}
$$

$I$ believe that the $\kappa_{l}$ are the natural tautological classes to consider on $\bar{M}_{g}$. On the other hand, the $\lambda_{l}$ are the natural classes for abelian varieties. Let me sketch this link, which will not be used subsequently. In fact, if

$$
A_{g}^{*}=\left(\begin{array}{l}
\text { Satake's compactification of the moduli } \\
\text { space of principally polarized abelian varieties }
\end{array}\right)
$$

then there is a natural morphism

$$
t: \bar{M}_{g} \longrightarrow A_{g}^{*}
$$

carrying the point [C] to the point of $A_{g}^{*}$ defined by the Jacobian of $C$. This morphism lifts to a $G$-equivariant morphism

$$
\tilde{t}: \tilde{\mathcal{M}}_{g} \rightarrow \tilde{A}_{g}
$$

where $\tilde{A}_{g}$ is a suitable toroidal compactification of the level $n$ covering of $\mathcal{A}_{g}$ : see Namikawa [N]. Moreover, $\tilde{A}_{g}$ carries a universal family

$$
\pi: \tilde{G}_{g} \longrightarrow \tilde{A}_{g}
$$

of semi-abelian group schemes, i.e., $\tilde{\mathscr{G}} / \tilde{A}$ is a group scheme whose fibres are extensions of abelian varieties by algcbraic tori $\left(\mathbb{C}^{*}\right)^{h}$. The family $\tilde{\mathscr{G}}_{g}$ pulls 
back on $\tilde{\mathcal{M}}_{g}$ to the family of Jacobians and generalized Jacobians of $\tilde{\mathcal{C}}_{g}$. Over $\tilde{A}_{g}$, define

$$
\begin{aligned}
& \mathbb{E}^{\prime}=\left.\Omega_{\tilde{C}_{o} / \tilde{\mathcal{A}}_{o}}^{1}\right|_{0-\text { section, }}, \text { a locally free sheaf of rank } g \\
& \lambda_{l}^{\prime}=c_{k}\left(\mathbb{E}^{\prime}\right), \quad 1 \leq l \leq g .
\end{aligned}
$$

Then it follows that

$$
\tilde{t}^{*} \mathbb{E}^{\prime} \cong \mathbb{E}
$$

and

$$
\tilde{t}^{*} \lambda_{l}^{\prime}=\lambda_{l}
$$

The class $K_{\overline{\mathcal{C}}_{0} / \overline{\mathcal{M}}_{o}}$ played a central role in the basic paper $[\Lambda]$ of $\Lambda$ rakelov, who proved the essential case of:

Theorem 4.1 (Arakelov). The divisor $K_{\bar{C}_{g} / \bar{M}_{\theta}}$ is numerically effective on $\bar{C}_{g}$, i.e., for all curves $C \subset \bar{C}_{g}$,

$$
\operatorname{deg}_{C} K_{\bar{C}_{o} / \overline{\mathcal{M}}_{o}} \geq 0
$$

Proof. In fact, Arakelov proved that for all normal surfaces $F$ fibred in stable curves over a smooth curve $C, \omega_{F / C}$ is ampı on $F$. This implies that for all curves $C \subset \tilde{C}_{g}$ such that $\pi(C) \cap \mathcal{M}_{g} \neq \phi$,

$$
\operatorname{deg}_{C} K_{\tilde{C}_{o} / \tilde{M}_{o}}>0
$$

Now suppose $C \subset \tilde{C}_{g}$ and $\pi(C) \subset \bar{M}_{g}-M_{g}$.

Case 1: $\pi(C)=$ one pt. Then $\operatorname{deg}_{C} K>0$ because $\omega$ is ample on all fibres of $\tilde{\mathcal{C}}_{g} \rightarrow \tilde{\mathcal{M}}_{g}$.

Case 2: $\left.d \pi\right|_{C} \equiv 0$, i.e., $C$ is in the locus Sing $C$ of double points of the fibres. But Sing $\mathcal{C}$ has an étale double cover Sing' $\mathcal{C}$ parametrizing pairs consisting of a double point of a fibre of $\pi$ and a branch through this point. By residue

$$
\omega_{\tilde{c}_{o} \mid \tilde{M}_{0}} \otimes O_{\text {Sing }^{\prime} C} \approx O_{\text {Sing }^{\prime} C}
$$

so $\operatorname{deg}_{C} K=\mathbf{0}$. 
Case 3: Other. After a suitable case change

$$
C^{\prime} \rightarrow \pi(C) \subset \tilde{\mathcal{M}}_{g}
$$

we can assume that the pull-back family $\tilde{C}_{g} \times_{\tilde{\mathcal{M}}_{g}} C^{\prime}$ is obtained by glueing several generically smooth stable families $Y_{\alpha} \rightarrow C^{\prime}$ along a set of sections $t_{\alpha \beta}: C^{\prime} \longrightarrow Y_{\alpha}$. Lying over $C$ there will be a curve $C^{\prime \prime}$ contained in one of the $Y_{\alpha}$ 's, say $Y_{\alpha_{0}}$, mapping onto $C^{\prime}$ and not equal to $t_{\alpha_{0} \beta}\left(C^{\prime}\right)$, any $\beta$. The pull-back of $\omega_{\tilde{\mathcal{C}}_{g} / \overline{\mathcal{M}}_{\alpha}}$ to $Y_{\alpha}$ will be equal to $\omega_{Y_{\alpha} / C^{\prime}}\left(\sum_{\beta} t_{\alpha_{0}, \beta}\left(C^{\prime \prime}\right)\right)$ and, by $\Lambda$ rakelov, this will have non-negative degree on $C^{\prime}$ if genus $C^{\prime} \geq 2$. If genus $C^{\prime}==0$ or 1 , it is easy to check that this is still the case.

Q.E.D.

Corollary 4.2. The classes $\kappa_{l}$ are numerically effective, i.e., for all subvarieties $W \subset \overline{\mathcal{M}}_{g}$ of dimension $l$,

$$
\left(W, \kappa_{l}\right) \geq 0
$$

Proof. $K_{\bar{C} / \bar{M}}$ numerically effective implies $K_{\overline{\mathcal{C}} / \overline{\mathcal{M}}}^{l+1}$ numerically effecitve (see $[\mathrm{K} 1])$, hence $\pi_{*}\left(K_{\overline{\mathcal{C}} / \bar{M}}^{l+1}\right)$ is numerically effective.

In fact, $\kappa_{1}$ is ample, sec $[\mathrm{M}], \S 5$.

\section{§5. Tautological Relations via Grothendicck-Ricmann-Roch}

Grothendieck's Riemann-Roch theorem (G-R-R) is, in many cases, tailormade to find relations among tautological classes. For example, see AtiyahBott $[\Lambda-B], \S 9$. We can compute the classes $\lambda_{k}$ in terms of the classes $\kappa_{k}$. To do this, we apply the G-R-R to the morphism

$$
\pi: \tilde{C}_{g} \longrightarrow \tilde{\mathcal{M}}_{g} \text {. }
$$

This gives us

$$
\operatorname{ch} \pi ! \omega_{\overline{\mathcal{C}} / \overline{\mathcal{M}}}=\pi_{*}\left(\operatorname{ch} \omega_{\overline{\bar{C}} / \overline{\mathcal{M}}} T d^{\vee}\left(\Omega \frac{1}{\bar{C} / \overline{\mathcal{M}}}\right)\right) .
$$

Here we use the notaion $T d^{\vee}(\mathcal{E})$ to write the universal multiplicative polynomial in the Chern classes of $\mathcal{E}$ such that for line bundles $L$,

$$
\begin{aligned}
T d^{\vee}(L) & =\frac{\lambda}{e^{\lambda}-1}, \quad \lambda=c_{1}(L) \\
& =1-\frac{1}{2} \lambda-\sum_{k=1}^{\infty}(-1)^{k-1} \frac{B_{k}}{(2 k) !} \lambda^{2 k}
\end{aligned}
$$


(i.e., the usual $T d(L)$ is $\lambda / 1-e^{-\lambda}$ or $1+\frac{1}{2} \lambda+\cdots$ ). Since $R^{1} \pi_{*} \omega_{\bar{C} / \overline{\mathcal{M}}} \cong O_{\overline{\mathcal{M}}}$, this means:

$$
\operatorname{ch} \mathbb{E}=1+\pi_{*}\left(e^{K} T d^{\vee}\left(\Omega_{\bar{C} / \bar{M}}^{1}\right)\right)
$$

Now use the exact sequence:

$$
0 \rightarrow \Omega \frac{1}{\mathcal{C} / \bar{M}} \rightarrow \omega_{\overline{\mathcal{C}} / \bar{M}} \rightarrow \omega_{\overline{\mathcal{C}} / \bar{M}} \otimes O_{\operatorname{Sing} \overline{\mathcal{C}}} \rightarrow 0
$$

(compare $[M]$, pf. of 5.10). Let Sing' $C$ be the double cover of Sing $C$ consisting of singular points plus branches: as a $Q$-variety, it is an étale double cover, i.e., the map between the charts

$$
\left(\operatorname{Sing}^{\prime} \bar{C}\right)_{\alpha} \rightarrow(\operatorname{Sing} \bar{C})_{\alpha}
$$

which are local universal deformation spaces, is étale. Then via residue

$$
\omega_{\bar{C} / \bar{M}} \otimes O_{\text {Sing }^{\prime} C} \cong O_{\text {Sing }} \bar{C} .
$$

Therefore:

$$
\begin{aligned}
\operatorname{ch} \mathbb{E} & =1+\pi_{*}\left(e^{K} \cdot T d^{\vee}\left(\omega_{\overline{\mathcal{C}} / \bar{M}}\right) \cdot T d^{\vee}\left(O_{\operatorname{Sing} \overline{\mathcal{C}}}\right)^{-1}\right) \\
& =1+\pi_{*}\left(e^{K} \cdot \frac{K}{e^{K}-1}+\left[T d^{\vee}\left(O_{\text {Sing } \bar{C}}\right)^{-1}-1\right]\right)
\end{aligned}
$$

since $K$ intersects any cycle on Sing $\bar{C}$ in zero. Now use the lemma:

Lemma 5.1. There is a universal power series $P$ such that for all $i: Z \rightarrow X$, an inclusion of a smooth codimension two subvariety in a smooth variety,

$$
\left(T d^{\vee} O_{Z}\right)^{-1}-1=i_{*}\left[P\left(c_{1} N, c_{2} N\right)\right]
$$

where $N$ is the normal bundle $I_{Z} / I_{Z}^{2}$.

Proof. In fact

$$
\left(T d^{\vee} O_{Z}\right)^{-1}=1 \dashv\left(\text { polyn. in } c h_{k}\left(O_{Z}\right), \quad k \geq 1\right)
$$

and by G-R-R for $i, c h_{k}\left(O_{Z}\right)$ is $i_{*}$ of a polynomial in $c_{1} N, c_{2} N$.

To compute this polynomial $P$, say $Z=D_{1} \cdot D_{2}$. Then use

$$
0 \rightarrow O_{X}\left(-D_{1}-D_{2}\right) \rightarrow O_{X}\left(-D_{1}\right) \oplus O_{X}\left(-D_{2}\right) \rightarrow O_{X} \rightarrow O_{Z} \rightarrow 0 .
$$


This gives us

$$
\begin{aligned}
T d^{\vee} O_{Z} & =\left(T d^{\vee} O_{X}\left(-D_{1}\right)\right)^{-1} \cdot\left(T d^{\vee} O_{X}\left(-D_{2}\right)\right)^{-1} \cdot T d^{\vee} O_{X}\left(-D_{1}-D_{2}\right) \\
& =\left(\frac{-D_{1}}{e^{-D_{1}}-1}\right)^{-1} \cdot\left(\frac{-D_{2}}{e^{-D_{3}}-1}\right)^{-1} \cdot\left(\frac{-D_{1}-D_{2}}{e^{-D_{1}-D_{2}}-1}\right) .
\end{aligned}
$$

Thus

$$
\begin{aligned}
D_{1} D_{2} \cdot P\left(D_{1}+D_{2}, D_{1} \cdot D_{2}\right)= & T d^{\vee}\left(O_{Z}\right)^{-1}-1 \\
= & \frac{D_{1}}{1-e^{-D_{1}}} \cdot \frac{D_{2}}{1-e^{-D_{2}}} \cdot \frac{1-e^{-D_{1}-D_{2}}}{D_{1}+D_{2}}-1 \\
= & \frac{1}{D_{1}+D_{2}} \cdot\left[D_{1} \cdot\left(\frac{D_{2}}{1-e^{-D_{2}}}-1\right)+\right. \\
& \left.D_{2} \cdot\left(\frac{D_{1}}{1-e^{-D_{1}}}-1\right)-D_{1} \cdot D_{2}\right] \\
= & \frac{D_{1} D_{2}}{D_{1}+D_{2}} \cdot \sum_{k=1} \frac{(-1)^{k-1} B_{k}}{(2 k) !}\left(D_{1}^{2 k-1}+D_{2}^{2 k-1}\right)
\end{aligned}
$$

So

$$
\begin{aligned}
P\left(D_{1}+D_{2}, D_{1} \cdot D_{2}\right)=\sum_{k=1}^{\infty} \frac{(-1)^{k-1} B_{k}}{(2 k) !}\left(\frac{D_{1}^{2 k-1}+D_{2}^{2 k-1}}{D_{1}+D_{2}}\right) \\
=\frac{1}{12}-\frac{1}{720}\left(\left(D_{1}+D_{2}\right)^{2}-3 D_{1} D_{2}\right)+ \\
\quad \frac{1}{30,240}\left(\left(D_{1}+D_{2}\right)^{4}-5 D_{1} D_{2}\left(D_{1}+D_{2}\right)^{2}+5 D_{1}^{2} D_{2}^{2}\right)+\cdots
\end{aligned}
$$

Therefore

$$
\operatorname{ch} \mathbb{E}=1+\pi_{*}\left(\frac{K}{1-e^{-K}}\right)+(\pi \circ i)_{*} P\left(c_{1} N, c_{2} N\right) .
$$

Now Sing $\bar{C}$ breaks up into pieces depending on whether the double point disconnects the fibre in which it lies or not, and if it does, what the genera are of the two pieces. Thus:

$$
\operatorname{Sing} \bar{C}=\coprod_{0 \leq h \leq[\boldsymbol{g} / 2]} \Delta_{h}^{*}
$$


where $\Delta_{0}^{*}$ are the non-disconnecting double points and if $h \geq 1, \Delta_{h}^{*}$ are the points for which one piece has genus $h$. Moreover, looking at the two pieces, one sees that

$$
\Delta_{h}^{*} \cong \bar{C}_{h} \times \bar{C}_{g-h} \text { if } 1<h<g / 2
$$

while

$$
\begin{aligned}
& \Delta_{g / 2}^{*} \cong \bar{C}_{g / 2} \times \bar{C}_{g / 2} /(\mathbb{Z} / 2 \mathbb{Z}) \text { if } g \text { is even } \\
& \Delta_{0}^{*} \cong \bar{M}_{g-1,2} /(\mathbb{Z} / 2 \mathbb{Z})
\end{aligned}
$$

where $\bar{M}_{g-1,2}$ is the space of stable curves with two ordered points $P_{1}, P_{2}$ and $\mathbb{Z} / 2 \mathbb{Z}$ permutes either the two factors or the two points. In fact, specifying a branch too, we get:

$$
\begin{aligned}
\text { Sing }^{\prime} \bar{C} & =\coprod_{0 \leq h \leq[g / 2]} \Delta_{h}^{\prime} \\
\Delta_{h}^{\prime} & \cong 2 \text { copies of } \bar{C}_{h} \times \bar{C}_{g-h} \quad 1 \leq h \leq g / 2 \\
& \cong \bar{C}_{g / 2} \times \bar{C}_{g / 2} \quad \text { if } h=g / 2 \\
& \cong \bar{M}_{g-1,2} \quad \text { if } h=0 .
\end{aligned}
$$

Let $K_{1}, K_{2}$ be the divisor classes defined

a) on $\bar{C}_{h} \times \bar{C}_{g-h}$ by $K_{1}=p_{1}^{*} K_{\bar{C}_{h} / \bar{M}_{h}}, K_{2}=p_{2}^{*} K_{\overline{\mathcal{C}}_{g-h} / M_{g-h}}$

b) on $\overline{\mathcal{M}}_{g-1,2}$ by $K_{i}=$ conormal bundle at the $i^{\text {th }}$ point.

Writing out $c h \mathbb{E}$ finally we get

$$
\begin{aligned}
\operatorname{ch} \mathbb{E}=g & +\sum_{l=1}^{\infty} \frac{(-1)^{l+1} \cdot B_{l}}{(2 l) !} . \\
& {\left[\kappa_{2 l+1}+\frac{1}{2} \sum_{h=0}^{g-1} i_{h, *}\left(K_{1}^{2 l-2}-K_{1}^{2 l-3} \cdot K_{2}+\cdots+K_{2}^{2 l-2}\right)\right] . }
\end{aligned}
$$

Ilere we have expanded $K / 1-e^{-K}$ and used the fact that $\pi_{*} K$ is $(2 g-2)$ times the fundamental class of $\overline{\mathcal{M}}_{g}$. The morphisin $i_{h}$ is

$$
\begin{aligned}
& i_{0}: \bar{M}_{g-1,2} \rightarrow \operatorname{Sing} \bar{C} \rightarrow \bar{M}_{g} \\
& i_{h}: \bar{C}_{h} \times \bar{C}_{g-h} \rightarrow \operatorname{Sing} \bar{C} \rightarrow \bar{M}_{g}, \quad 1 \leq h \leq g-1 .
\end{aligned}
$$


Note that $i_{0}$ and $i_{g / 2}$ have degree 2 and the other $i_{h}$ 's are repeated twice in the sum: hence the factor $1 / 2$. Moreover, we have evaluated the normal bundle to Sing $\bar{C}$ in $\bar{C}_{g}$ as the direct sum of the tangent bundle to the two branches of the curve at the singular point:

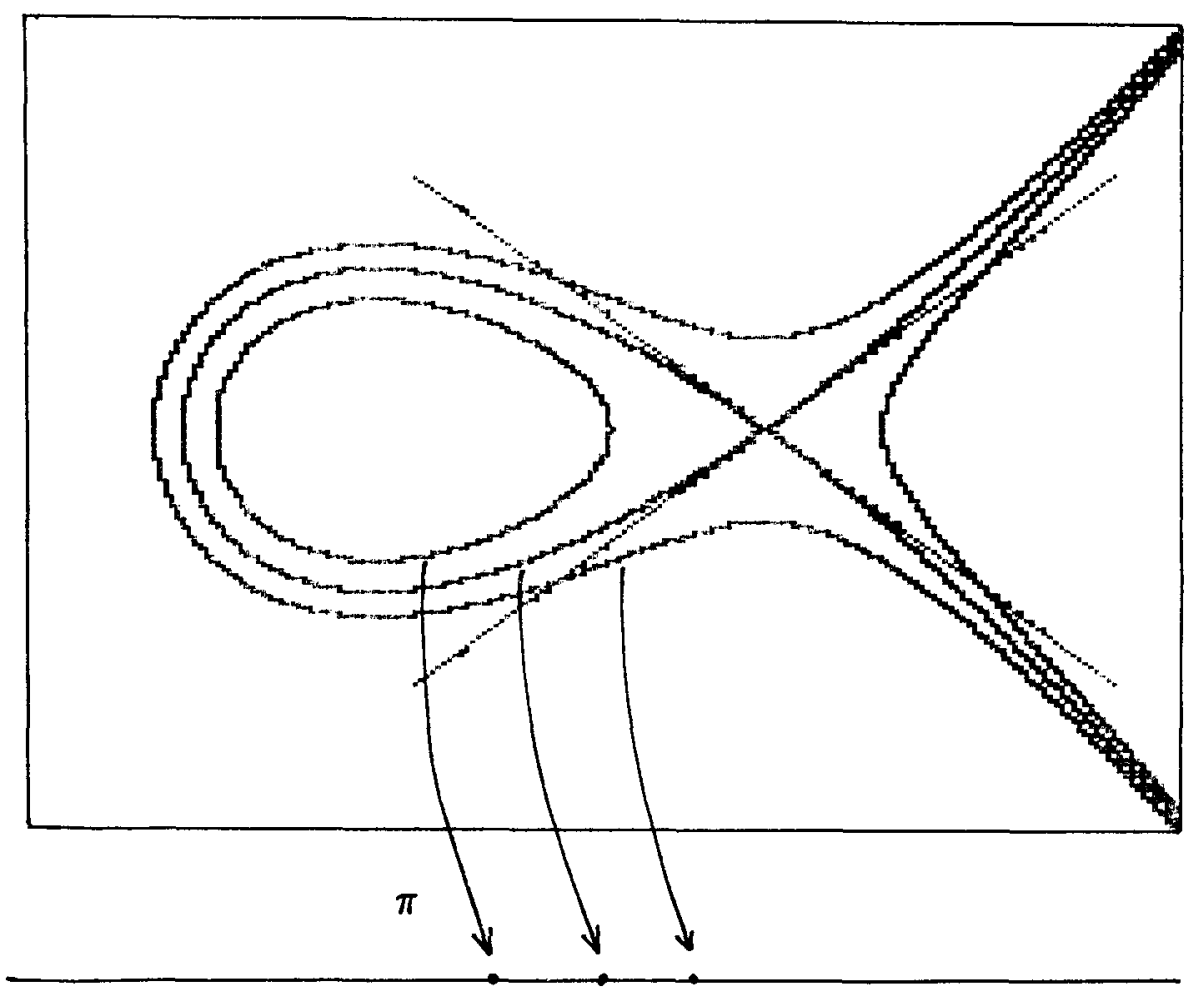

(In a transversal to Sing $\bar{C}, \bar{C}_{g} / \overline{\mathcal{M}}_{g}$ looks like $x y=t$, and the tangent bundle to the $x, y$-surface at $(0,0)$ is the surn of the tangent line to the branch $x=0$ and to the branch $y=0$.)

The formula (5.2) specializes in codimension 1 to the formula of $[\mathrm{M}]$, p. 102: 


$$
\lambda_{1}=c_{l}(\mathbb{E})=\frac{1}{12}\left(\kappa_{1}+\delta\right)
$$

where

$$
\begin{aligned}
\delta & =\frac{1}{2} \sum_{h=0}^{g-1} i_{h, *}(1) \\
& =\text { fundamental class of } \bar{M}_{g}-\mathcal{M}_{g} .
\end{aligned}
$$

Moreover, it proves

Corollary (5.3). For all even integers $2 k$,

$$
(\operatorname{ch} \mathbb{E})_{2 k}=\mathbf{0} \text {. }
$$

This formula can be proven in cohomology from the Gauss-Manin connection. We sketch this proof. First look at the smooth curves $C_{g} / M_{g}$. For these we have the DeRham complex

$$
\Omega_{C_{g} / M_{g}}: 0 \rightarrow O_{C_{g}} \stackrel{d}{\rightarrow} \Omega_{C_{g} / M_{g}}^{1} \rightarrow 0
$$

along the fibres of $\pi$. This gives:

$$
0 \rightarrow \pi_{*} \Omega_{C_{g} / M_{g}}^{1} \rightarrow \mathbb{R}^{1} \pi_{*} \Omega_{C_{g} / M_{g}} \rightarrow R^{1} \pi_{*} O_{C_{g}} \rightarrow 0 .
$$

By Serre duality, this gives:

$$
0 \rightarrow \mathbb{E} \rightarrow \mathbb{R}^{1} \pi_{*} \Omega_{\mathcal{C}_{g} / \mathcal{M}_{g}} \rightarrow \mathbb{E}^{\vee} \rightarrow 0 .
$$

The vector bundle in the middle has rank $2 g$, is isomorphic to $R^{1} \pi_{*} \mathbb{C}$ and possesses the Gauss-Manin connection. Therefore its Chern classes are zero and over $\mathcal{M}_{g}$ :

$$
c(\mathbb{E}) \cdot c\left(\mathbb{E}^{\vee}\right)=1 .
$$

This identity can be extended to $\overline{\mathcal{M}}_{g}$ if we use the complex

$$
\omega_{{\overline{C_{g}}}_{9} / \bar{M}_{\theta}}: 0 \rightarrow O_{\mathcal{C}_{\theta}} \stackrel{d}{\rightarrow} \omega_{\bar{C}_{\theta} / \bar{M}_{\theta}} \rightarrow 0
$$


from which we get the sequence:

$$
0 \rightarrow \mathbb{E} \rightarrow \mathbb{R}^{1} \pi_{*} \omega_{\overline{\mathcal{C}}_{g} / \bar{M}_{\theta}} \rightarrow \mathbb{E}^{\vee} \rightarrow 0 .
$$

Although the Gauss-Manin connection does not extend regularly to $\mathbb{R}^{1} \pi_{*} \omega_{\mathcal{C}_{g} / \bar{M}_{g}}^{\cdot}$, it has regular singularities with a polar part which is nilpotent. This is enough to conclude that its Chern classes zero, extending (5.5) to $\bar{M}_{g}$. This means equivalently that

$$
\operatorname{ch}(\mathbb{E})+\operatorname{ch}\left(\mathbb{E}^{\vee}\right)=0
$$

or

$$
\operatorname{ch}(\mathbb{E})_{2 k}=0, \quad k \geq 1 .
$$

This identity in fact holds on $\tilde{A}_{g}$, the toroidal compactification of $\mathcal{A}_{g}$. It can be deduced, for instance, from the extension of Hirzebruch's proportionality theorern to $\tilde{A}_{g}$ (see $[\mathrm{M} 2]$ ).

The conclusion to be drawn from (5.2) and (5.3) is that the even $\lambda_{k}$ 's are polynomials in the odd ones, and that all the $\lambda_{k}$ 's are polynomials in the $\kappa_{k}$ 's and in boundary cycles. Moreover, applying (5.2) in odd degree above $g$, we can express $\kappa_{k}$ for $k$ odd, $k>g$, in terms of lower $\kappa_{l}$ 's and boundary cycles. We shall strengthen this in the next section, where we find a simpler way to get identities on the $\kappa_{k}$ 's.

The exact sequence (5.4) is remarkable in another way that reveals something of the nature of $\bar{M}_{g}$. Note that $\mathbb{E}$ tends to be a positive bundle: at least $c_{1}(\mathbb{E})$ is the pull-back of an ample line bundle by a birational map. But it is also a sub-bundle of a bundle with connection, i.e., the DeRharn bundle $\mathbb{R}^{1} \pi_{*} \omega^{\cdot}$ is unstable yet lias a connection.

\section{§6. Tautological Relations via the Canonical Linear System}

There is another very different way to get relations on the $\lambda_{i}$ and $\kappa_{i}$. For this, we will not try to get the full relations in $A^{\cdot}\left(\overline{\mathcal{M}}_{g}\right)$ as the boundary terms seem to be a bit involved, but instead get the relations in $A^{\prime}\left(M_{g}\right)$. Because of the exact sequence:

$$
A^{\prime}\left(\overline{\mathcal{M}}_{g}-\mathcal{M}_{g}\right) \rightarrow A^{\prime}\left(\overline{\mathcal{M}}_{g}\right) \rightarrow A^{\prime}\left(\mathcal{M}_{g}\right) \rightarrow 0
$$

this is the same as a relation in $A \cdot\left(\overline{\mathcal{M}}_{\theta}\right)$ with an undeternined boundary term. 
The method is based on the fact that for all smooth curves $C$, the sheaf $\omega_{C}$ is generated by its global sections. ${ }^{5}$

Now if we let $\tilde{C}_{g} / \tilde{M}_{g}$ temporarily stand for the family of smooth stable curves, i.e., replace $\tilde{C}_{g}$ by $\pi^{-1}\left(\mathcal{M}_{g}\right)$, then we have an exact sequence:

$$
0 \rightarrow \mathcal{F} \rightarrow \pi^{*} \pi_{*} \omega_{\tilde{\mathcal{C}}_{g} / \tilde{\mathcal{M}}_{g}} \rightarrow \omega_{\tilde{\mathcal{C}}_{g} / \tilde{\mathcal{M}}_{\mathrm{g}} \rightarrow 0}
$$

where all these sheaves are $Q$-sheaves and $\mathcal{F}$ is locally free of rank $g-1$. Taking Chern classes, we get:

$$
c(\mathcal{F})=\pi^{*}\left(1+\lambda_{1}+\cdots+\lambda_{g}\right) \cdot\left(1+K_{\check{C}_{0} / \tilde{M}_{g}}\right)^{-1} .
$$

Using the fact that $c_{n}(\mathcal{F})=0$ if $n \geq g$, this says:

$$
\left(K_{\tilde{C} / \tilde{M}}^{n}\right)-\pi^{*}\left(\lambda_{1}\right) \cdot\left(K_{\tilde{C} / \tilde{M}}^{n-1}\right)+\cdots+(\dot{-}-1)^{g} \pi^{*}\left(\lambda_{g}\right) \cdot\left(K_{\tilde{C} / \tilde{M}}^{n-g}\right)=0
$$

for all $n \geq g$. Taking $\pi_{*}$, this means

$$
\begin{array}{rll}
\kappa_{n-1}-\lambda_{1} \cdot \kappa_{n-2}+\cdots+(-1)^{g} \lambda_{g} \cdot \kappa_{n-g-1} & =0 & \text { if } n \geq g+2 \\
\kappa_{g}-\lambda_{1} \cdot \kappa_{g-1}+\cdots+(-1)^{g} \lambda_{g} \cdot(2 g-2) & =0 & \text { if } n=g+1 \\
\kappa_{g-1}-\lambda_{1} \cdot \kappa_{g-2}+\cdots+(-1)^{g-1} \lambda_{g-1} \cdot(2 g-2)=0 & \text { if } n=g .
\end{array}
$$

${ }^{5}$ If $C$ is a singular stable curve, then one can show that $\Gamma\left(\omega_{C}\right)$ generates the subsheaf of $\omega_{C}$ of sections which are zero

i) at all double points $P$ for which $C-P$ is disconnectd,

ii) on all components $E_{0}$ of $C$ which are isomorphic to $P^{1}$ and such that all double

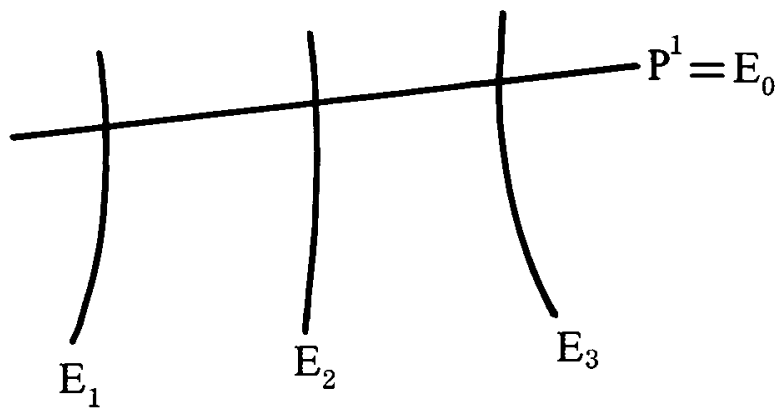

points $P$ on $E_{0}$ are disconnecting domble points. 
Corollary 6.2. For all $g$, all the classes $\lambda_{i}, \kappa_{i}$ restricted to $A^{\prime}\left(\mathcal{M}_{g}\right)$ are polynomials in $\kappa_{1}, \kappa_{2}, \ldots, \kappa_{g-2}$.

Proof. This is clear except for $\kappa_{g}, \kappa_{g-1}$. Here we must combine the above relations with (5.2). There are two cases depending on whether $g$ is even or odd. Recalling that

$$
c h_{n} \mathbb{E}=\frac{(-1)^{n-1} \cdot c_{n}(\mathbb{E})}{(n-1) !}+\text { polyn. in lower Chern class }
$$

it follows that if $g=2 k$ or $2 k-1$, then

$$
\left.\frac{(-1)^{k+1} \cdot B_{k}}{(2 k) !} \kappa_{2 k-1}=c h_{2 k-1} \mathbb{E}=\frac{\lambda_{2 k-1}}{(2 k-2) !}+\text { (polyn. in lower } \lambda \text { 's }\right) \text {. }
$$

If $g=2 k$, we want to show that the 2 equations

$$
\left\{\begin{aligned}
(-1)^{k+1} B_{k} \cdot \kappa_{2 k-1} & =2 k \cdot(2 k-1) \lambda_{2 k-1}+\text { lower } \\
\kappa_{2 k-1} & =2 \cdot(2 k-1) \lambda_{2 k-1}+\text { lower }
\end{aligned}\right.
$$

have independent leading terms, and if $g=2 k-1$, then we want to do the same with

$$
\left\{\begin{aligned}
(-1)^{k+1} B_{k} \cdot \kappa_{2 k-1} & =2 k \cdot(2 k-1) \lambda_{2 k-1}+\text { lower } \\
\kappa_{2 k-1} & =4(k-1) \cdot \lambda_{2 k-1}+\text { lower. }
\end{aligned}\right.
$$

This follows, however, by inspection if $k \leq 10$ and for larger $k$ by the estimate:

$$
\begin{array}{r}
B_{k}=\frac{2 \cdot(2 k) !}{(2 \pi)^{2 k}} \zeta(2 k)>\frac{2 \cdot(2 k / e)^{2 k}}{(2 \pi)^{2 k}}=2 \cdot\left(\frac{k}{e \cdot \pi}\right)^{2 k}>2 \cdot k \text { if } k \geq 11 . \\
\text { Q.E.D. }
\end{array}
$$

With this approach, the first relation between $\kappa_{1}, \ldots, \kappa_{g-2}$ that we get occurs in codimension $g+1$ or $g+2$. One should, however, get the relation $\kappa_{1}^{2}=0$ in $A \cdot\left(M_{3}\right)$ so we clearly do not have all the relations on the $\kappa_{i}$ 's and $\lambda_{i}$ 's yet. It does seem reasonable to conjecture, however, that $\kappa_{1}, \ldots, \kappa_{g-2}$ have no relations up to something like codimension $g$, e.g., $g-($ small constant). 
§7. The Tautological Classes via Arbarello's Flag of Subvarieties of $\mathcal{M}_{g}$ We want to consider the following subsets of $C_{g}$ and $\mathcal{M}_{g}$ :

$$
\begin{aligned}
W_{l}^{*} & =\left\{C, x \in C_{g} \mid h^{o}\left(C_{C}(l \cdot x)\right) \geq 2\right\} \\
& =\left\{\begin{array}{cc}
C, x \in C_{g} \mid \exists \text { a morphism } \pi: c \rightarrow \mathbb{P}^{1} \text { of degree } \\
d \leq l \text { with } \pi^{-1}(\infty)=d . x
\end{array}\right\} \\
W_{l} & =\pi\left(W_{l}^{*}\right) \subset \mathcal{M}_{g}
\end{aligned}
$$

where $2 \leq l \leq g$. Thus $W_{g}^{*}=$ locus of Weierstrass points in $C_{g}$, $W_{g-1}=$ curves with an exceptional Weierstrass of one of the two simplest types, and $W_{2}=$ hyperelliptic curves. Note that:

$$
\begin{aligned}
& \mathcal{C}_{g} \supset W_{g}^{*} \supset W_{g-1}^{*} \supset \cdots \supset W_{2}^{*} \\
& \mathcal{M}_{g}=W_{g} \supset W_{g-1} \supset \cdots \supset W_{2} .
\end{aligned}
$$

I first heard of this flag from E. Arbarello who proposed (see [Arb $]^{6}$ ) that they might be used as a ladder to climb from the reasonably well-known space $W_{2}$ to the still mysterious $\mathcal{M}_{g}$.

Let me first recall and sketch the proof of the following well-known facts:

Proposition 7.1. $\quad W_{l}^{*}$ is irreducible of codimension $g-l+1$ and $W_{l}^{*}-W_{l-1}^{*}$ is an open dense subset smooth in the local charts for $C_{g}$, i.e., the local deformation space for the pairs $(C, x)$.

Sketch of proof. Firstly, $W_{l}^{*}$ is a determinantal subvaricty of $\tilde{C}_{g}$. In fact, consider

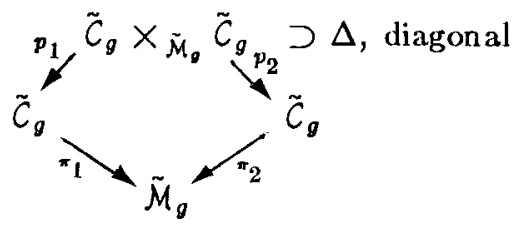

Let

$$
\mathcal{F}_{l}=R^{1} p_{2, *}\left(\mathcal{O}_{\tilde{c} \times \tilde{c}}(l \Delta)\right)
$$

Then over a point $[C, x] \in \tilde{C}_{g}$,

$$
\mathcal{F}_{l} \otimes \mathbf{k}([C, x]) \cong H^{1}\left(C, O_{C}(l . x)\right)
$$

\footnotetext{
${ }^{6}$ Unfortunately, the proof of Theorem 3.27 in $[\Lambda \mathrm{rb}]$ is incomplete as it stands.
} 
Now if $[C, x] \notin W_{l}^{*}, h^{0}\left(O_{C}(l x)\right)=1$ and $h^{1}\left(O_{C}(l x)\right)=g-l$, while if $[C, x] \in W_{l}^{*}$, both numbers are bigger. Thus $\mathcal{F}_{l}$ is locally free of rank $g-l$ on $\tilde{C}_{g}-W_{l}^{*}$ and not locally free anywhere on $W_{l}^{*}$. But look at the sequence:

$$
0 \rightarrow O_{\tilde{c} \times \tilde{c}} \rightarrow O_{\tilde{c} \times \tilde{c}}(l \Delta) \rightarrow o_{\tilde{c} \times \tilde{c}}(l \Delta) / O_{\tilde{c} \times \tilde{c}} \rightarrow 0
$$

which gives us:

$$
0 \rightarrow p_{2, *}\left(O_{\tilde{C} \times \tilde{C}}(l \Delta) / O_{\tilde{C} \times \tilde{C}}\right) \stackrel{\alpha}{\rightarrow} R^{1} p_{2, *}\left(O_{\tilde{C} \times \tilde{C}}\right) \rightarrow \mathcal{F}_{l} \rightarrow 0 .
$$

The first sheaf is locally free of rank $l$, the second locally free of rank $g$, hence

$$
W_{l}^{*}=\left\{[C, x] \in \tilde{C}_{g} \mid r k_{[C, x]}(\alpha)<l\right\} .
$$

Thus the codimension of $W_{l}^{*}$ is at most $g-l+1$. But describing $W_{l}^{*}-W_{l-1}^{*}$ as the set of $l$-fold covers of $\mathbb{P}^{1}$, totally ramified at $\infty$, one gets the upper bound $2 g+l_{1}-3$ on $\operatorname{dim} W_{l_{1}}^{*}-W_{l_{1}-1}^{*}$ for all $l_{1}$, hence the same upper bound on $\operatorname{dim} W_{l}^{*}$. Comparing the two, it follows that $\operatorname{codim} W_{l}^{*}$ is exactly $g-l+1$ and $W_{l}^{*}$ is determinantal as well as that $W_{l}^{*}-W_{l-1}^{*}$ is dense in $W_{l}^{*}$. The irreducibility of $W_{l}^{*}-W_{l-1}^{*}$ is a classical result of Lüroth, describing all $l$-fold covers of $\mathbb{P}^{1}$ as brariched covers with a standard set of transpositions.

The smoothness of $W_{l}^{*}-W_{l-1}^{*}$ in the universal deformation space may be checked by the following calculation: let $f$ have an $l$-fold pole at $x \in C$ and make an infinitesimal deformation $C$ of $C$ over $\mathbb{C}[\epsilon]$ by glueing open sets $U_{\alpha} \times \operatorname{Spec} \mathbb{C}[\epsilon]$ via a 1-cocycle $D_{\alpha \beta}$ of derivations zero at $x$. Then $f$ lifts to a rational function on $C$ with $l$-fold pole at $x$ if there are functions $g_{\alpha}$ with $l$-fold poles at $x$ and:

$$
\left(1+\epsilon D_{\alpha \beta}\right)\left(f+\epsilon g_{\alpha}\right)=f+\epsilon g_{\beta} .
$$

This means that $\left\{D_{\alpha \beta} f\right\} \in H^{1}(C, O(l x))$ is zero. But $D_{\alpha \beta} f=\left\langle D_{\alpha \beta}, d f\right\rangle$ is the image:

$$
\left\{D_{\alpha \beta}\right\} \in H^{1}\left(C, T_{C}(-x)\right) \underset{(, d f)}{\longrightarrow} H^{1}\left(C, O_{C}(l x)\right) .
$$

Note that $H^{1}\left(C, T_{C}(-x)\right)$ is the tangent space to the universal deformation space of $(C, x)$. Moreover (7.3) is dual to the injeclive map

$$
I^{0}\left(C, O\left(2 K_{C}+x\right)\right) \underset{\otimes d f}{\leftarrow} H^{0}\left(C, O\left(K_{C}-l x\right)\right)
$$


hence (7.3) is surjective, i.e., the subscheme of the universal deformation space where $f$ lifts is smooth of codimension $h^{1}\left(O_{C}(l x)\right)=g-l$.

In order to work out the fundamental class of $W_{l}^{*}$, it is convenient to split up (7.2) into pieces as follows. Starting with

$$
\begin{array}{r}
0 \rightarrow O_{\tilde{c} \times \tilde{c}}((l-1) \Delta) \rightarrow O_{\tilde{c} \times \tilde{c}}(l \Delta) \rightarrow O_{\tilde{c} \times \tilde{c}}(l \Delta) / O_{\tilde{c} \times \tilde{c}}((l-1) \Delta) \rightarrow 0 \\
\| \\
O_{\Delta} \otimes p_{2}^{*} O_{\tilde{c}}\left(-l K_{\tilde{c} / \tilde{\mathcal{M}}}\right)
\end{array}
$$

we get via $R^{*} p_{2, *}$ :

$$
0 \rightarrow O_{\tilde{C}}\left(-l K_{\tilde{C} / \tilde{M}}\right) \stackrel{\beta}{\rightarrow} \mathcal{F}_{l-1} \rightarrow \mathcal{F}_{l} \rightarrow 0 .
$$

It follows that on $\tilde{C}_{g}-W_{l-1}^{*}$ where $\tilde{F}_{l-1}$ is locally free:

$$
\begin{aligned}
W_{l}^{*} & =\left\{[C, x] \in \tilde{C}_{g} \mid \beta_{[C, x]}=0\right\} \\
& =\text { zeroes of the section } \beta^{\prime} \in \Gamma\left(\tilde{C}_{g}, \mathcal{F}_{l-1}\left(l K_{\tilde{C} / \tilde{\mathcal{M}}}\right)\right) .
\end{aligned}
$$

Moreover, on the universal deformation space of $[C, x]$, this section $\beta^{\prime}$ vanishes to $1^{\text {st }}$ order along $W_{l}^{*}$ : in fact, the differential of $\beta^{\prime}$ at a point of $W_{l}^{*}$ is a map

$$
\begin{gathered}
T_{[C, x], \mathcal{C}_{\alpha}} \longrightarrow \mathcal{F}_{l-1}(l k) \otimes \mathrm{k}([C, x]) \\
\|
\end{gathered}
$$

which is readily seen to be the surjective map (7.3) (the factor $\left(\mathcal{M}_{x} / \mathcal{M}_{x}^{2}\right)^{l}$ is hidden in (7.3) in the choice of $f$ ). Thus

$$
\left[W_{l}^{*}\right]_{Q}=c_{g-l+1}\left(F_{l-1}\left(l K_{\tilde{C} / \tilde{\mathcal{M}}}\right)\right), \quad \text { on } \tilde{C}_{g}-W_{l-1}^{*} \text {. }
$$

But $W_{l-1}^{*}$ has codimension $g-l+2$ so

$$
A^{g-l+1}\left(C_{g}\right) \cong A^{g-l+1}\left(C_{g}-W_{l-1}^{*}\right) .
$$

Thus (7.5) holds as an equation in $A^{g-l+1}\left(C_{g}\right)$, hence in op $A^{g-1+1}\left(\tilde{C}_{g}\right)$. Now let's calculate the fundamental class of $W_{i}^{*}$ :

$$
\begin{aligned}
{\left[W_{l}^{*}\right]_{Q} } & =c_{g-l+1}\left(\mathcal{F}_{l-1}(l K)\right) \\
& =c_{g-l+1}\left(\mathcal{F}_{l}(l K)\right) \\
& =c_{g-l+1}\left(\mathcal{F}_{l}\right) .
\end{aligned}
$$


Here we have abbreviated $K_{\tilde{\mathcal{C}} / \tilde{\mathcal{M}}}$ to $K$, and the last equality follows from the general fact

$$
\begin{aligned}
& c_{n}(\mathcal{G}(D))= \\
& \quad c_{n}(\mathcal{G})+(r-n+1) D \cdot c_{n-1}(\mathcal{G})+\left(\begin{array}{c}
r-n+2 \\
2
\end{array}\right) D^{2} \cdot c_{n-2}(\mathcal{G})+\cdots+\left(\begin{array}{l}
r \\
n
\end{array}\right) \cdot D^{n}
\end{aligned}
$$

$(r=$ generic rank 9$)$, whence

$$
c_{r+1}(\mathcal{G}(D))=c_{r+1}(\mathcal{G}), \text { all divisors } D .
$$

But now

$$
\begin{aligned}
c\left(\mathcal{F}_{l}\right)= & c\left(\mathcal{F}_{l-1}\right) \cdot(1-l K)^{-1} \\
= & c\left(\mathcal{F}_{l-2}\right) \cdot(1-(l-1) K)^{-1}(1-l K)^{-1} \\
& \cdots \\
= & c\left(\mathcal{F}_{0}\right) \cdot(1-K)^{-1} \cdot(1-2 K)^{-1} \cdot \cdots \cdot(l-l K)^{-1} \\
= & \pi_{2}^{*}\left(c\left(R^{1} \pi_{1}, * O_{\tilde{C} / \tilde{\mathcal{M}}}\right)\right) \cdot(1-K)^{-1} \cdots \cdot(1-l K)^{-1} \\
= & \pi_{2}^{*}\left(1-\lambda_{1}+\lambda_{2}-\cdots+(-1)^{g} \lambda_{g}\right) \cdot(1-K)^{-1} \cdots \cdot(1-l K)^{-1}
\end{aligned}
$$

'Thus

$$
\begin{aligned}
{\left[W_{l}^{*}\right]_{Q}=} & (g-l+1)^{s t} \text { component of } \\
& \pi^{*}\left(1-\lambda_{1}+\lambda_{2}-\cdots+(-1)^{g} \lambda_{g}\right) \cdot(1-K)^{-1} \cdots(1-l K)^{-1}
\end{aligned}
$$

If we define $W_{l}$ as a cycle as $\pi_{*}\left(W_{l}^{*}\right)$, we get also

$$
\begin{aligned}
{\left[W_{l}\right]_{Q}=} & (g-l)^{t h} \text {-component of } \\
& \left(1-\lambda_{1}+\lambda_{2}-\cdots+(-1)^{g} \lambda_{g}\right) \pi_{*}\left[(1-K)^{-1} \cdot \cdots \cdot(1-l K)^{-1}\right] .
\end{aligned}
$$

This shows that $\left[W_{l}\right]_{Q}$ is a polynomial in the tautological classes $\kappa_{j}$. Presumably the coefficient of $\kappa_{l}$ is always non-zero and hence we can solve for the $\kappa_{l}$ 's in terms of the classes $\left[W_{j}\right]$, but this looks like a messy calculation.

Let's work out the hyperelliptic locus $\mathcal{H}$ as an example. Note that

$$
W_{2}^{*} \rightarrow W_{2}
$$

is a covering of degree $2 g+2$ because for all hyperelliptic curves $C$, there are exactly $2 g+2$ points $x$ such that $h^{\circ}\left(O_{C}(2 x)\right) \geq 2$-- namely the Wcierstrass 
points. Thus

$$
\begin{aligned}
{[K]_{Q}=} & \frac{1}{2 g+2}\left[W_{2}\right]_{Q} \\
= & \frac{1}{2 g+2}\left\{\begin{array}{l}
(g-2)^{n d} \text { component of } \\
\left(1-\lambda_{1}+\cdots+(-1)^{g} \lambda_{g}\right) \cdot \pi_{*}\left((1-K)^{-1} \cdot(1-2 K)^{-1}\right)
\end{array}\right\} \\
= & \frac{1}{2 g+2}\left\{\left(2^{g}-1\right) \kappa_{g-2}-\left(2^{g-1}-1\right) \lambda_{1} \cdot \kappa_{g-3}+\cdots+\right. \\
& \left.(-1)^{g-3} \cdot 7 \cdot \lambda_{g-3} \cdot \kappa_{3}+(-1)^{g-2} \cdot(6 g-6) \lambda_{g-2}\right\} .
\end{aligned}
$$

Finally every hyperelliptic curve has an automorphism of order 2, so

$$
\begin{aligned}
{[\mathcal{H}] } & =2 \cdot[\mathcal{H}]_{Q} \\
& =\frac{1}{g+1}\left\{\left(2^{g}-1\right) \kappa_{g-2}-\cdots+(-1)^{g-2}(6 g-2) \lambda_{g-2}\right\} .
\end{aligned}
$$

\section{Part III: The Case $\mathrm{g}=2$}

§8. Tautological Relations in Genus 2

First of all, let's specialize the calculations of Part II to the case $g=2$ and see what we have. From $\mathbb{E}$, we get 2 elements

$$
\begin{aligned}
& \lambda_{1} \in A^{1}\left(\bar{M}_{2}\right) \\
& \lambda_{2} \in A^{2}\left(\bar{M}_{2}\right)
\end{aligned}
$$

and because $c h_{2}(\mathbb{E})=0$, we get

$$
\lambda_{2}=\lambda_{1}^{2} / 2
$$

From $K$ on $\bar{C}_{2}$, we get

$$
\kappa_{i} \in A^{i}\left(\bar{M}_{2}\right), \quad i=1,2,3 .
$$

The calculations of $\S 5$ give us the relation:

$$
\lambda_{1}=\frac{1}{12}\left(\kappa_{1}+\delta\right)
$$


Here $\bar{M}_{2}-M_{2}$ has 2 components $\Delta_{0}$ and $\Delta_{1}, \Delta_{0}$ the closure of the locus of irreducible singular curves, $\Delta_{1}$ the locus of singular curves $C_{1} \cup C_{2}$, $C_{1} \cap C_{2}=$ one pt., $p_{a}\left(C_{1}\right)=p_{1}\left(C_{2}\right)=1$. By definition

$$
\delta=\left[\Delta_{0}\right]_{Q}+\left[\Delta_{1}\right]_{Q} .
$$

We shall write $\delta_{0}$ for $\left[\Delta_{0}\right]_{Q}$ and $\delta_{1}$ for $\left[\Delta_{1}\right]_{Q}$. We don't need (5.2) in codimension 3 but it provides an interesting check on the calculations later. It gives:

$$
\frac{1}{6} \lambda_{1}^{3}-\frac{1}{2} \lambda_{1} \lambda_{2}=c h_{3} \mathbb{E}=-\frac{1}{720}\left[\kappa_{3}+\frac{1}{2} \cdot \sum_{h=0}^{1} i_{h, *}\left(\left(K_{1}+K_{2}\right)^{2}-3 K_{1} \cdot K_{2}\right)\right]
$$

or

$$
60 \lambda_{1}^{3}=\kappa_{3}+\frac{1}{2} \sum_{h=0}^{1} i_{h, *}\left(K_{1}^{2}-K_{1} K_{2}+K_{2}^{2}\right) .
$$

In $\S 10 b$ we shall work out these terms numerically and check this.

We can refine the calculations of $\$ 6$ by working out the boundary term too. It is easy to see that if $C$ is a stable curve of genus $2, \omega_{C}$ is generated by its global sections, unless $C=C_{1} \cup C_{2}, C_{1} \cap C_{2}=\{P\}$, in which case $\Gamma\left(\omega_{C}\right)$ generates $m_{P} \cdot \omega_{C}$. Therefore, working over the whole of $\tilde{C}_{2}$ we get:

$$
0 \rightarrow \mathcal{F} \rightarrow \pi^{*} \pi_{*} \omega_{\tilde{C}_{2} / \tilde{\mathcal{M}}_{2}} \rightarrow I_{\Delta_{1}^{*}} \cdot \omega_{\tilde{C}_{3} / \tilde{\mathcal{M}}_{2}} \rightarrow 0
$$

(following the notation of $\S 5$ ). $I_{\Delta_{i}^{*}}$ has two generators at every point, so its projective dimension is 1 , i.e., $\mathcal{F}$ is locally free, hence invertible. Now use:

$$
0 \rightarrow I_{\Delta_{i}} \cdot \omega \rightarrow \omega \rightarrow \omega \otimes O_{\Delta_{i}^{*}} \rightarrow 0
$$

and the fact that via residue, $\omega$ is trivial on the double cover $\Delta_{1}^{\prime}$ of $\Delta_{1}^{*}$, hence $\omega^{2}$ is trivial on $\Delta_{1}^{*}$. It follows that

$$
c(\mathcal{F})=\pi^{*}\left(1+\lambda_{1}+\lambda_{2}\right) \cdot\left(1+K_{\tilde{C}_{2} / \tilde{\mathcal{M}}_{2}}\right)^{-1} \cdot c\left(O_{\Delta_{1}^{*}}\right) .
$$

A useful lemma that we can use here is:

Lemma 8.2. If $Y \subset X$ is a local complete intersection of codimension 2, and $i: Y \rightarrow X$ is the inclusion, then

$$
c\left(O_{Y}\right)=1-i_{*}\left(c\left(I_{Y} / I_{Y}^{2}\right)^{-1}\right) .
$$


Proof. If $Y=D_{1} . D_{2}$ globally, then this formula is easily checked. But by the G-R-R,

$$
c\left(O_{Y}\right)=1+i_{*}\left(\text { univ. polyn. in } c_{1}\left(I / I^{2}\right), c_{2}\left(I / I^{2}\right)\right)
$$

and the universal polynomial must be $c\left(I / I^{2}\right)^{-1}$ because the two are equal whenever $Y==D_{1}, D_{2}$.

As in $\S 5, \Delta_{1}^{\prime} \cong \bar{M}_{1,1} \times \bar{M}_{1,1}$, i.e., there is a $2-1$ map:

$$
i_{1}: \bar{M}_{1,1} \times \bar{M}_{1,1} \rightarrow \Delta_{1}^{*}
$$

and $i_{1}^{*}\left(I / I^{2}\right)=K_{1}+K_{2}$. Thus

$$
c\left(O_{\Delta_{i}}\right)=1-\frac{1}{2} \cdot i_{1, *}\left(\left(1-K_{1}-K_{2}\right)^{-1}\right)
$$

Thus

$$
\begin{aligned}
c(\mathcal{F})= & \pi^{*}\left(1+\lambda_{1}+\lambda_{2}\right) \cdot \\
& \left(1-K+K^{2}-K^{3}+K^{4}\right) \cdot\left(1-\frac{1}{2} \cdot i_{1, *}\left(1+K_{1}+K_{2}+K_{1} \cdot K_{2}\right)\right) .
\end{aligned}
$$

In particular,

$$
\mathbf{0}=c_{2}(\mathcal{F})=\pi^{*} \lambda_{2}-K . \pi^{*} \lambda_{1}+K^{2}-\left[\Delta_{1}^{*}\right]_{\ell}
$$

Multiplying this by $K$ and $K^{2}$, we get even simpler formulae:

$$
\begin{aligned}
& 0=K \cdot \pi^{*} \lambda_{2}-K^{2} \cdot \pi^{*} \lambda_{1}+K^{3} \\
& 0=K^{2} \cdot \pi^{*} \lambda_{2}-K^{3} \cdot \pi^{*} \lambda_{1}+K^{4}
\end{aligned}
$$

Taking $\pi_{*}$, this gives

$$
\begin{aligned}
& \kappa_{1}=2 \lambda_{1}+\delta_{1} \\
& \kappa_{2}=\kappa_{1} \cdot \lambda_{1}-2 \lambda_{2}=\lambda_{1} \cdot\left(\lambda_{1}+\delta_{1}\right) \\
& \kappa_{3}=\kappa_{2} \lambda_{1}-\kappa_{1} \lambda_{2}=\frac{1}{2} \lambda_{1}^{2}\left(\delta_{1}\right) .
\end{aligned}
$$


Combining (8.1) and (8.3), we see that both $\kappa_{1}$ and $\lambda_{1}$ are expressible in terms of $\delta_{0}, \delta_{1}$ :

$$
\begin{aligned}
& 10 \lambda_{1}=\delta_{0}+2 \delta_{1} \\
& 5 \kappa_{1}=\delta_{0}+7 \delta_{1} .
\end{aligned}
$$

As $\kappa_{1}$ is ample, this implies the well-known fact that $\mathcal{M}_{2}$ is affine!

This relation (8.4) has a very simple analy tic proof. Consider the modular form of weight 10 on Sicgel's space $H_{2}$ given by

$$
f(Z)=\left[\prod_{a, b \text { even }} \theta\left[\begin{array}{l}
a \\
b
\end{array}\right](0, Z)\right]^{2}
$$

(Each $\theta$ has weight $1 / 2$ and there are ten even $a, b$ 's.) It vanishes on $\mathcal{H}_{2}$ precisely when

$$
\gamma Z=\left(\begin{array}{ll}
Z_{1} & 0 \\
0 & Z_{2}
\end{array}\right), \quad \text { some } \gamma \in S p(4, \mathbb{Z})
$$

and then to order 2. At the principal cusp

$$
\left(\begin{array}{ll}
i \infty & w \\
w & z
\end{array}\right)
$$

it has the form

$$
\begin{aligned}
& \text { (unit) } \cdot \theta\left[\begin{array}{ll}
1 & 0 \\
0 & 0
\end{array}\right]^{2} \cdot \theta\left[\begin{array}{ll}
1 & 1 \\
0 & 0
\end{array}\right]^{2} \cdot \theta\left[\begin{array}{ll}
1 & 0 \\
0 & 1
\end{array}\right]^{2} \cdot \theta\left[\begin{array}{ll}
1 & 1 \\
1 & 1
\end{array}\right]^{2} \\
= & \left(\text { unit) } \cdot\left(e^{\pi i(1 / 2) \Omega_{11}}\right)^{8}\right. \\
= & \text { unit } \cdot e^{2 \pi i \Omega_{11}}
\end{aligned}
$$

i.e., it vanishes to order 1 . Thus $f$ defines a section of $\left(\Lambda^{2} \mathbb{E}\right)^{\otimes 10}$ whose zeroes in $\tilde{\mathcal{M}}_{2}$ are $2 \tilde{\Delta}_{1}+\tilde{\Delta}_{0}$. This reproves (8.4).

§9. Generators of $A \cdot\left(\bar{M}_{2}\right)$.

We use the exact sequence:

$$
A .(Y) \rightarrow A .(X) \rightarrow A .(X-Y) \rightarrow 0
$$


( $Y \subset X$ closed subvariety) to get generators of $A .\left(\bar{M}_{2}\right)$. Recall that $M_{2}$ is known from Igusa's results [I] to be isomorphic to $\mathbb{C}^{3}$ modulo $\mathbb{Z} / 5 \mathbb{Z}$ acting by

$$
(x, y, z) \mapsto\left(\varsigma x, \varsigma^{2} x, \varsigma^{3} y\right) .
$$

Then

$$
A .\left(\mathbb{C}^{3}\right) \rightarrow A \cdot\left(\mathcal{M}_{2}\right)
$$

is surjective, hence $A_{k}\left(M_{2}\right)=(0)$, if $k<3$. Thus

$$
A_{k}\left(\Delta_{0}\right) \oplus A_{k}\left(\Delta_{1}\right) \rightarrow A_{k}\left(\bar{M}_{2}\right)
$$

is surjective if $k<3$. In particular, $A_{2}\left(\bar{M}_{2}\right)$ is generated by $\delta_{0}$ and $\delta_{1}$.

Define the dimension 1 subsets:

$$
\begin{aligned}
\Delta_{00}= & \left\{\begin{array}{l}
\text { closure of curve in } \bar{M}_{2} \text { parametrizing } \\
\text { irreducible rational curves with } 2 \text { nodes }
\end{array}\right\} \\
\Delta_{01}= & \Delta_{0} \cap \Delta_{1} \\
= & \text { Curve in } \bar{M}_{2} \text { parametrizing curves } C_{0} \cup C_{2}, \\
& \text { where } C_{1} \cap C_{2}=\{x\}, C_{1} \text { is elliptic or } \\
& \text { rational with one node and } \\
& C_{2} \text { is rational with one node }
\end{aligned}
$$

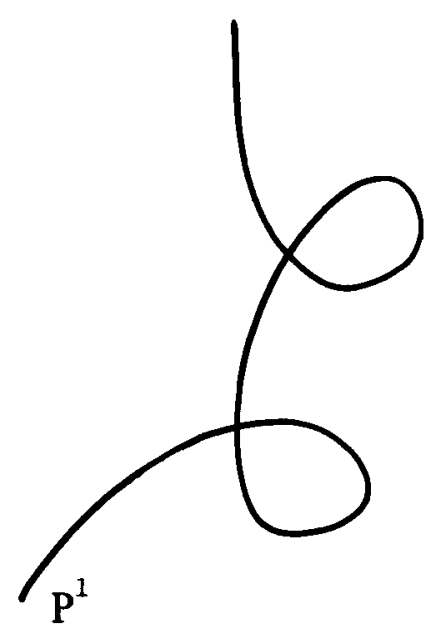

curves in $\Delta_{00}$

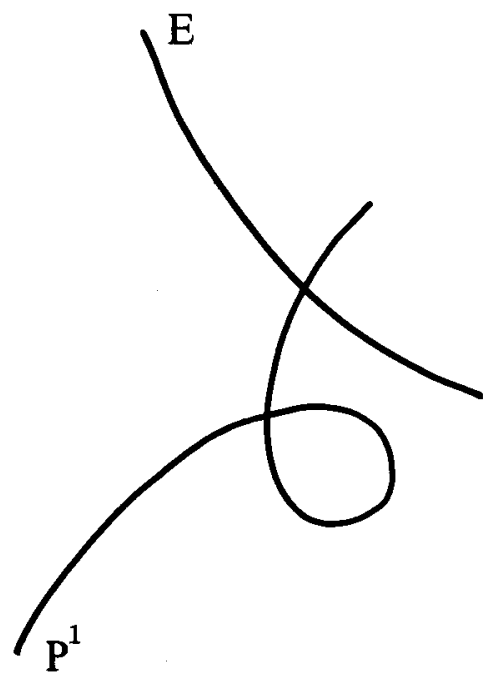

curves in $\Delta_{01}$ 
Note that $\Delta_{00}$ contains, besides the irreducible curves illustrated, the two reducible curves

\section{$C_{000}$ :}
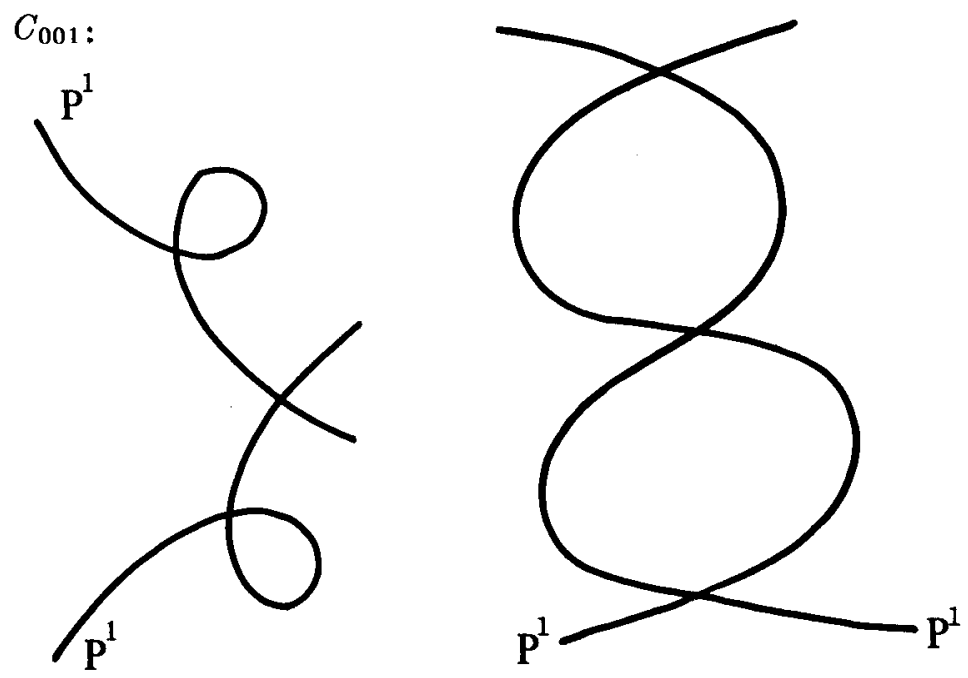

Int $\Delta_{1}=\left(\Delta_{1}-\Delta_{01}\right)$ is the locus of curves $C_{1} \cup C_{2}$ where $C_{1} \cap C_{2}=\{x\}$, $C_{1}, C_{2}$ sinooth elliptic. It is isomorphic to $\operatorname{Symm}^{2} \mathcal{M}_{1,1}$, i.e., to the product of the affine $j$-line by itself mod the involution interchanging the factors. Therefore it is coordinatized by $j\left(C_{1}\right)+j\left(C_{2}\right), j\left(C_{1}\right) \cdot j\left(C_{2}\right)$ :

$$
\text { Int } \Delta_{1} \cong \mathbb{C}^{2} \text {. }
$$

Moreover, Int $\Delta_{0}=\Delta_{0}-\left(\Delta_{00} \cup \Delta_{01}\right)$ is the locus of irreducible elliptic curves with one node, i.e., the space $\mathcal{M}_{1,2}$ of triples $\left(E, x_{1}, x_{2}\right), x_{1}, x_{2} \in E$, $x_{1} \neq x_{2} \bmod$ the involution intercharging the 2 points. Write all elliptic curves as

$$
y^{2}=x(x-1)(x-\lambda), \quad \lambda \neq 0,1
$$

and take $x_{1}=$ pt. at $\infty, x_{2}=(x, y)$. Interchanging $x_{1}, x_{2}$ carries $(x, y)$ to $(x,-y)$. So we get a surjective map

$$
\{(x, \lambda) \mid \lambda \neq 0,1\} \rightarrow \operatorname{Int} \Delta_{0} .
$$


Putting this together

$$
\begin{aligned}
& A_{k}\left(\Delta_{1}-\Delta_{01}\right)=(0), \quad k<2 \\
& A_{k}\left(\Delta_{0}-\left(\Delta_{00} \cup \Delta_{01}\right)\right)=(0), \quad k<2 .
\end{aligned}
$$

Thus $A_{1}\left(\bar{M}_{2}\right)$ is generated by:

$$
\delta_{00}=\left[\Delta_{00}\right]_{Q}
$$

and

$$
\delta_{01}=\left[\Delta_{01}\right]_{Q}
$$

Pinally, $\bar{M}_{2}$ is unirational so all points of $\bar{M}_{2}$ are rationally equivalent and we have proven:

Proposition 9.1. $\quad \bar{M}_{2}$ is the disjoint union of 7 cells:

$$
\bar{M}_{2}=\mathcal{M}_{2} \coprod \operatorname{Int} \Delta_{0} \coprod \operatorname{Int} \Delta_{1} \coprod \operatorname{Int} \Delta_{00} \coprod \operatorname{Int} \Delta_{01} \coprod\left\{C_{000}\right\} \coprod\left\{C_{001}\right\} .
$$

Correspondingly, $A \cdot\left(\overline{\mathcal{M}}_{2}\right)$ is generated by

a) 1 in codimension 0 ,

b) $\delta_{0}, \delta_{1}$ in codimension 1 ,

c) $\delta_{00}, \delta_{01}$ in codimension 2 ,

d) the class $[x]$ of a point in codimension 3: call this $p$.

Note that by the results of $\S 8, \lambda_{1}$ and $\kappa_{1}$ are also generators in codimension 1 . We shall see that all the above cycles are independent. This will follow as a Corollary once we work out the multiplication table for these cycles.

§10. Multiplication in $A^{\cdot}\left(\bar{M}_{2}\right)$

We shall prove:

Theorem 10.1. The ring $A\left(\overline{\mathcal{M}}_{2}\right)$ has a $\mathbb{Q}$-basis consisting of $1, \delta_{0}$, 
$\delta_{1}, \delta_{00}, \delta_{01}, p$ and multiplication table:

$$
\begin{aligned}
\delta_{0}^{2} & =\frac{5}{3} \delta_{00}-2 \delta_{01} \\
\delta_{0} \cdot \delta_{1} & =\delta_{01} \\
\delta_{1}^{2} & =-\frac{1}{12} \delta_{01} \\
\delta_{0} \cdot \delta_{00} & =-\frac{1}{4} p \\
\delta_{0} \cdot \delta_{01} & =\frac{1}{4} p \\
\delta_{1} \cdot \delta_{00} & =-\frac{1}{8} p \\
\delta_{1} \cdot \delta_{01} & =-\frac{1}{48} p
\end{aligned}
$$

An easier way to describe the ring structure is via $\lambda_{1}$. Using the identities $10 \lambda_{1}=\delta_{0}+2 \delta_{1}$, we can describe the multiplication by:
a) $\delta_{0} \cdot \delta_{1}=\delta_{01}$
b) $\delta_{00} \cdot \delta_{1}=\frac{1}{8} p$
c) $\delta_{00} \cdot \lambda_{1}=0$
d) $\delta_{1} \cdot \lambda_{1}=\frac{1}{12} \delta_{01}$
e) $\delta_{0} \cdot \lambda_{1}=\frac{1}{6} \delta_{00}$

The reader can check that these are equivalent to the relations of the Theorem. 
Relations (a) and (b) are proper intersections of cycles and are proved by the explicit formula of $\S 3$ : thus (a) follows because the lifts of $\delta_{0}, \delta_{1}$ to the universal deformation space of a curve $C \in \Delta_{01}$ are smooth divisors meeting transversely in the smooth curve lifting $\Delta_{01}$. And for (b), $\Delta_{00} \cap \Delta_{1}$ is the one curve $C_{001}$ whose automorphism group has order 8 . In the universal deformation space of $C_{001}, \Delta_{00}$ and $\Delta_{1}$ lift to a smooth curve and surface meeting transversely, so

$$
\delta_{00} . \delta_{1}=\left[C_{001}\right]_{Q}=\frac{1}{8} p
$$

c) is an immediate consequence of the general theory of Knudsen [K] or of the fact that $\delta_{00}$ is blown down to a point in the Satake compactification $A_{2}^{*}$ of $A_{2}$. To prove (d), consider

$$
i_{1}: \bar{M}_{1,1} \times \bar{M}_{1,1} \rightarrow \Delta_{1} \subset \bar{M}_{2} .
$$

We check that $i_{1}^{*}\left(\lambda_{1}-\frac{1}{12} \delta_{0}\right)=0$, hence $\left(\lambda_{1}-\frac{1}{12} \delta_{0}\right) \cdot \delta_{1}=0$. But

$$
i_{1}^{*}\left(\lambda_{1}^{(2)}\right)=p_{1}^{*}\left(\lambda_{1}^{(1)}\right)+p_{2}^{*}\left(\lambda_{1}^{(1)}\right)
$$

where, for the sake of clarity, we write

$$
\begin{aligned}
& \lambda_{1}^{(2)}=\text { the class } \lambda_{1} \text { in } A^{\cdot}\left(\overline{\mathcal{M}}_{2}\right) \\
& \lambda_{1}^{(1)}=\text { the class } \lambda_{1} \text { in } A^{\cdot}\left(\overline{\mathcal{M}}_{1,1}\right) .
\end{aligned}
$$

This is simply because on curves $E_{1} \cup E_{2}, E_{i}$ elliptic, $E_{1} \cap E_{2}=$ one point,

$$
\Gamma\left(E_{1} \cup E_{2}, \omega_{E_{1} \cup E_{2}}\right) \cong \Gamma\left(E_{1}, \omega_{E_{1}}\right) \oplus \Gamma\left(E_{2}, \omega_{E_{2}}\right) .
$$

Moreover,

$$
i_{1}^{*}\left(\delta_{0}^{(2)}\right)=p_{1}^{*}\left(\delta^{(1)}\right)+p_{1}^{*}\left(\delta^{(1)}\right) .
$$

But in $A \cdot\left(\bar{M}_{1,1}\right)$, the relation

$$
\lambda_{1}=\frac{1}{12} \delta
$$

holds. This is well known and is just the specialization to genus 1 of the theory of $\S 5$. Or else it may be seen using the elliptic modular form $\Delta$ of 
weight 12 with a simple pole at the cusp. Finally, to prove (e), consider:

$$
i_{0}: \bar{M}_{1,2} \rightarrow \Delta_{0} \subset \bar{M}_{2}
$$

Then $i_{0, *}\left(1_{\bar{M}_{1,2}}\right)=2 \delta_{0}$. One should be careful here to note that the presence of automorphisms generically on $\bar{M}_{2}$ does not affect this: in fact

$$
\begin{aligned}
i_{0, *}\left(1_{\overline{\mathcal{M}}_{1,2}}\right) & =i_{0, *}\left(\left[\overline{\mathcal{M}}_{1,2}\right]\right) \\
& =\left[\Delta_{0}\right] \quad \text { (because } \overline{\mathcal{M}}_{1,2} \rightarrow \Delta_{0} \text { is birational) } \\
& =2 \delta_{0} \quad \text { (because } \operatorname{Aut}(C)=\mathbb{Z} / 2 \mathbb{Z},[C] \in \Delta_{0} \text { generic) }
\end{aligned}
$$

Therefore

$$
\begin{aligned}
\lambda_{1} \cdot \dot{\delta}_{0} & =\frac{1}{2} \lambda_{1} \cdot i_{0, *}\left(1_{\overline{\mathcal{M}}_{2,1}}\right) \\
& =\frac{1}{2} i_{0, *}\left(i_{0}^{*}\left(\lambda_{1}\right)\right) .
\end{aligned}
$$

Now let

$$
\pi: \bar{M}_{1,2} \longrightarrow \bar{M}_{1,1}
$$

be the natural projection. Note that $\bar{M}_{1,1}$ is the $j$-line and $\bar{M}_{1,2}$ is the universal family over the $j$-line of elliptic curves mod automorphisms. Then

$$
i_{0}^{*}\left(\lambda_{1}^{(2)}\right)=\pi^{*}\left(\lambda_{1}^{(1)}\right)
$$

by Knudsen's theory. This corresponds to the fact that if $E^{\prime}$ is elliptic with one node $P$, and $E$ is the normalization of $E^{\prime}$, then there is a canonical sequence

$$
\mathbf{0} \rightarrow \Gamma\left(\omega_{E}\right) \rightarrow \Gamma\left(\omega_{E^{\prime}}\right) \stackrel{\text { res }}{\rightarrow} \mathbf{k}(P) \rightarrow \mathbf{0}
$$

hence $\Lambda^{2}\left(\Gamma\left(\omega_{E^{\prime}}\right)\right) \cong \Gamma\left(\omega_{E}\right)$. Therefore

$$
\begin{aligned}
\lambda_{1} . \delta_{0} & =\frac{1}{2} i_{0, *}\left(\pi^{*}\left(\lambda_{1}^{(1)}\right)\right) \\
& =\frac{1}{24} i_{0, *}\left(\pi^{*}\left(\delta^{(1)}\right)\right) .
\end{aligned}
$$

But $\pi^{*}\left(\delta^{(1)}\right)=[\tilde{\Delta}],[\tilde{\Delta}] \subset \bar{M}_{1,2}$ being the closure of the locus of triples $\left(C, x_{1}, x_{2}\right), C$ a rational curve with a node, $x_{1}, x_{2}$ distinct smooth points of $C . \tilde{\Delta}$ maps birationally to $\Delta_{00}$ in $\bar{M}_{2}$, and the automorphism group of 
the generic rational curve with 2 nodes is $(\mathbb{Z} / 2 \mathbb{Z})^{2}$, hence:

$$
\begin{aligned}
\lambda_{1} \cdot \delta_{0} & =\frac{1}{24}\left[\Delta_{00}\right] \\
& =\frac{1}{6} \delta_{00} .
\end{aligned}
$$

\section{$\S 10 b$. A Check}

An interesting check that these $Q$-stack-theoretic calculations are $O K$ is to evaluate all terms in the identity

$$
60 \lambda_{1}^{3}=\kappa_{3}+\frac{1}{2} \sum_{h=0}^{1} i_{h, *}\left(K_{1}^{2}-K_{1} K_{2}+K_{2}^{2}\right)
$$

obtained in $§ 8$. Using Theorem 10.1 , one finds

$$
60 \lambda_{1}^{3}=\frac{1}{48} p
$$

Using (8.3) plus theorem 10.1 , one finds

$$
\kappa_{3}=\frac{1}{1152} p
$$

To calculate

$$
i_{0, *}\left(K_{1}^{2}-K_{1} K_{2}+K_{2}^{2}\right)
$$

let $\pi: \bar{M}_{1,2} \rightarrow \bar{M}_{1,1}$ be the natural map and in $\tilde{\mathcal{M}}_{1,2}$ consider the points $\pi^{-1}([E])$, i.e., representing $\left(E, x_{1}, x_{2}\right)$, with $E$ fixed. Up to automorphisms of $E, x_{1}$ can be normalized to be the identity. Letting $x_{2}$ vary, we parametrize this subset of $\tilde{\mathcal{M}}_{1,2}$ by $E$ itself, and describe the universal family of triples $\left(E, x_{1}, x_{2}\right)$ as $E \times E$ over $E$ with $x_{1}$ being given by $s_{1}(x)=e(x), x_{2}$ by the diagonal $s_{2}(x)=(x, x)$ : 


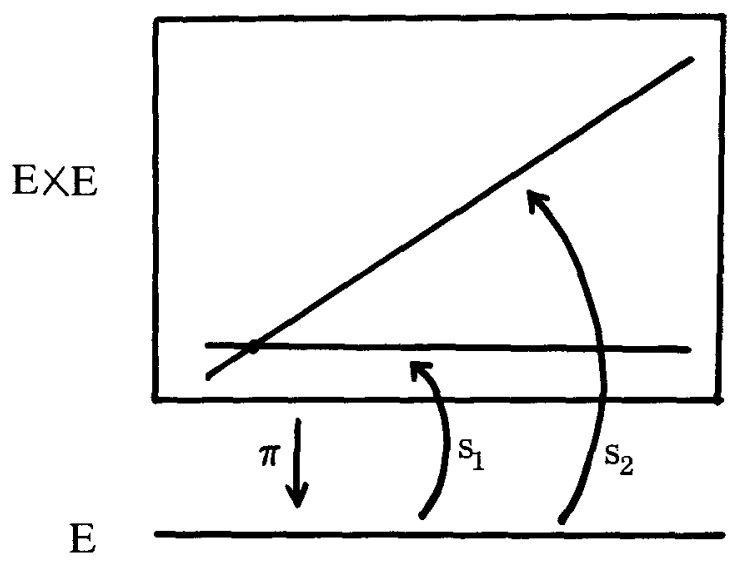

However, this allows $x_{1}=x_{2}$ over $e \in E$, where we should have instead $E \cup \mathbb{P}^{1}, x_{1}, x_{2} \in \mathbb{P}^{1}-E \cap \mathbb{P}^{1}, x_{1} \neq x_{2}$. Thus we must blow up $(e, e) \in E \times E$, getting:

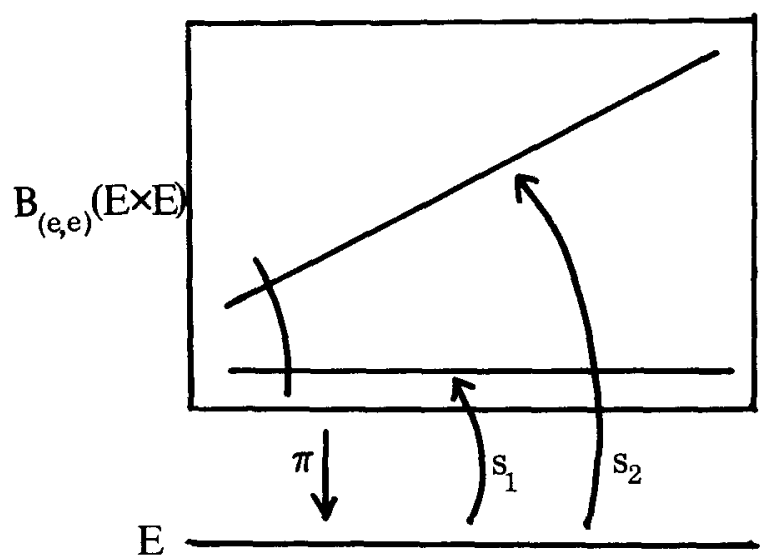


This is now a farnily of 2-pointed stable clliptic curves. The conormal bundle $K_{i}$ to $s_{i}$ is $\mathcal{O}_{E}(+e)$. Thus on $\bar{M}_{1,2}$, the invertible $Q$-sheaf $O\left(K_{i}\right)$, restricted to the fibres over $\bar{M}_{1,1}$, is $O_{\overline{\mathcal{M}}_{1,2}}\left(+\Sigma_{1}\right)$ where $\Sigma_{1} \subset \bar{M}_{1,2}$ is the locus of 2-pointed stable curves:

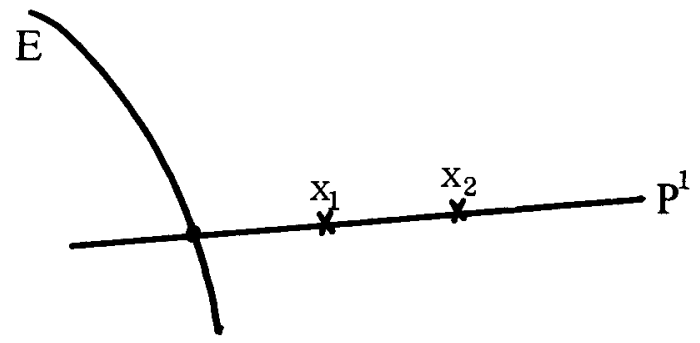

Therefore $K_{1} \equiv K_{2} \equiv \pi^{*}(A)+\Sigma_{1}$, for some $Q$-divisor class $A$ on $\bar{M}_{1,1}$. But along $\sum_{1}$, a canonical coordinate can be put on $\mathbb{P}^{1}$ making $E \cap \mathbb{P}^{1}=\{\infty\}, x_{1}=0, x_{2}=1$, hence $\mathcal{O}_{\bar{M}_{1,2}}\left(K_{i}\right)$, restricted to $\Sigma_{1}$, is trivial. Now $\pi^{*} O_{\bar{M}_{1,1}}\left(\lambda_{1}\right) \cong \omega_{\bar{M}_{2,1} / \bar{M}_{1,1}}$, hence the conormal bundle to $\Sigma_{1}$ is $O\left(\lambda_{1}\right)$. This proves:

$$
K_{1} \equiv K_{2} \equiv \pi^{*}\left(\lambda_{1}\right)+\Sigma_{1}
$$

Therefore

$$
\begin{aligned}
K_{1}^{2}-K_{1} K_{2}+K_{2}^{2} & =\pi^{*} \lambda_{1} \cdot \Sigma_{1} \\
& =\frac{1}{12} \pi^{*} \delta . \Sigma_{1} \\
& =\frac{1}{24} p
\end{aligned}
$$

hence

$$
\frac{1}{2} i_{0, *}\left(K_{1}^{2}-K_{1} K_{2}+K_{2}^{2}\right)=\frac{1}{48} p .
$$

Finally to calculate

$$
i_{1, *}\left(K_{1}^{2}-K_{1} K_{2}+K_{2}^{2}\right)
$$


note that on $\bar{M}_{1,1} \times \bar{M}_{1,1}, K_{1}^{2}=K_{2}^{2}=0$, and $K_{i}$ is the pull back from $\bar{M}_{1,1}$ of $\lambda_{1}$. Since $\lambda_{1}=\frac{1}{24} p$,

$$
\begin{aligned}
\frac{1}{2} i_{1, *}\left(K_{1}^{2}-K_{1} K_{2}+K_{2}^{2}\right) & =\frac{1}{2} i_{1, *}\left(-\frac{1}{24} \cdot \frac{1}{24} p\right) \\
& =-\frac{1}{1152} p .
\end{aligned}
$$

This checks!

\section{References}

[A] Arakelov, S., Families of algebraic curves with fixed degeneracies, Izv. Akad. Nauk, 35 (1971).

[Arb] Arbarello, E., Weierstrass points and moduli of curves, Comp. Math., 29 (1974), pp. 325-342.

[A-B] Atiyah, M., and Bott, R., The Yang-Mills equations over Riemann surfaces, to appear.

[B-F-M] Baum, P., Fulton, W., and MacPherson, R., Riemann-Roch for singular varieties, Publ. I.H.E.S., 45 (1975), pp. 101-145.

[D-M] Deligne, P., and Mumford, D., The irreducibility of the space of curves of given genus, Publ. I.H.E.S. 36 (1969) pp. 75-109.

[F1] Fulton, W., Rational equivalence on singular varieties, Publ. I.H.E.S. 45 (1975), pp. 147-167.

[F2] Fulton, W., Intersection Theory, Springer-Verlag, 1983.

[F-M] Fulton, W. and MacPherson, R., Categorical framework for the study of singular spaces, Memoirs A.M.S. 243, (1981).

[Ha] Harer, J., The second homology group of the mapping class group of an orientable surface, to appear.

[H-M] Harris, J., and Mumford, D., On the Kodaira dimension of the moduli space of curves, Inv. Math., 67 (1982), pp. 23-86.

[H] Hironaka, H., Bimeromorphic smoothing of a complex-analytic space, Acta. Math. Vietnamica, 2(1977), pp. 103-168.

[1] Igusa, J.I., Arithmetic theory of moduli for genus two, Annals of Math., 72 (1960), pp. 612-649.

[K] Knudsen, F., The projectivity of the moduli space of stable curves, Math. Scand. to appear. 
[K1] Kleiman, S., Towards a numerical theory of ampleness, Annals of Math., 84 (1966), pp. 293-344.

[Ma] Matsusaka, T., Theory of Q-varieties, Publ. Math. Soc. Japan, 8 (1964)

[Mi] Miller, E., The homology of the mapping class group of surfaces, in preparation.

[M] Mumford, D., Stability of projective varieties, L'Ens, Math., 24 (1977), pp. 39-110.

[M2] Mumford, D., Hirzebruch's proportionality principle in the noncompact case, Inv. math., 保 (1977), pp. 239-272.

[N] Namikawa, Y., A new compactification of the Siegel space and degeneration of abelian varieties $I$ and $I I$, Math. Annalen, 221 (1976), pp. 97--141, 201-241.

Received July 1, 1982

Professor David Mumford

Department of Mathematics

Harvard University

Cambridge, Massachusetts 02138 